\title{
Lost \& found dark matter in elliptical galaxies
}

\author{
A. Dekel ${ }^{1,2,3}$, F. Stoehr ${ }^{2}$, G.A. Mamon ${ }^{2}$, T.J. Cox ${ }^{4}$, G.S. Novak ${ }^{5}$, \& J.R. \\ Primack $^{3}$ \\ ${ }^{1}$ Racah Institute of Physics, The Hebrew University, Jerusalem 91904, Israel \\ ${ }^{2}$ Institut d'Astrophysique and Observatoire de Paris, 98bis Boulevard Arago, Paris 75014, France \\ ${ }^{3}$ Department of Physics, University of California, Santa Cruz CA 95064, USA \\ ${ }^{4}$ Center for Astrophysics, Harvard University, 60 Garden Street, Cambridge MA 02138, USA \\ ${ }^{5} \mathrm{UCO} /$ Lick Observatories, University of California, Santa Cruz CA 95064, USA
}

There is strong evidence that the mass in the Universe is dominated by dark matter, which exerts gravitational attraction but whose exact nature is unknown. In particular, all galaxies are believed to be embedded in massive haloes of dark matter. ${ }^{1,2}$ This view has recently been challenged by surprisingly low random stellar velocities in the outskirts of ordinary elliptical galaxies, which were interpreted as indicating a lack of dark matter. ${ }^{3,4}$ Here we show that the low velocities are in fact compatible with galaxy formation in darkmatter haloes. Using numerical simulations of disc-galaxy mergers, ${ }^{5,6}$ we find that the stellar orbits in the outer regions of the resulting ellipticals are very elongated. These stars were torn by tidal forces from their original galaxies during the first close passage and put on outgoing trajectories. The elongated orbits, combined with the steeply falling density profile of the observed tracers, explain the observed low velocities even in the presence of large amounts of dark matter. Projection effects when viewing a triaxial elliptical can lead to even lower observed velocities along certain lines of sight.

\section{Introduction}

The common spiral galaxies are known to reside in extended dark-matter (DM) haloes. The rotational speeds of their gas discs do not decline outside the visible body, ${ }^{1}$ unlike the expectation from Keplerian circular velocities at a radius $r$ about a mass $M, V^{2}=$ $G M / r$. Thus, the DM mass within $r$ is growing roughly as $M(r) \propto r$ and it dominates the gravitational potential beyond a certain radius. An extrapolation based on the typical halo density profile ${ }^{7}$ found in simulations of the standard $\Lambda$ CDM cosmology predicts an outer "virial" radius $R_{\text {vir }}$ that is 50-100 times larger than the characteristic stellar radius, enclosing 10-20 times more DM than luminous matter, now also indicated observationally.. ${ }^{8}$ 
The conventional wisdom is that the potential wells created by the DM are crucial for seeding the formation of galaxies. ${ }^{2,9,10}$

The standard hypothesis is that ellipticals originate from mergers of discs ${ }^{11}$ and should therefore be embedded in similar DM haloes. There is evidence for DM in giant ellipticals, from X-rays ${ }^{12}$ and gravitational lensing ${ }^{13}$ and in nearby dwarf galaxies from stellar kinematics. ${ }^{14}$ However, ordinary ellipticals lack obvious velocity tracers at the large projected radii $r_{\mathrm{p}}$ where the DM is expected to be important. This is typically beyond $R_{\text {eff }},{ }^{15}$ the "effective" radius encompassing half the projected light, while measurements of the projected velocity dispersion $\sigma_{\mathrm{p}}$ of the stellar light are limited to $r_{\mathrm{p}}<2 R_{\mathrm{eff}}$.

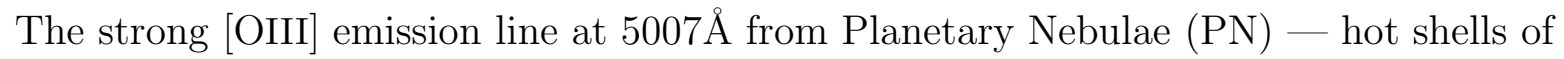
gas expelled from dying stars of $(1-3) M_{\odot}$ - provide a unique tool for extracting $\sigma_{\mathrm{p}}\left(r_{\mathrm{p}}\right)$ beyond $R_{\text {eff }}$. Romanowsky et al. ${ }^{4}$ measured $\sigma_{\mathrm{p}}$ from $\sim 100$ bright PNs in each of three normal ellipticals, NGC 821, 3379 and 4494, adding to 531 PNs in NGC 4697 by Mendez et al.. ${ }^{3}$ They find that $\sigma_{\mathrm{p}}$ typically drops by a factor $\simeq 1.6$ between $r_{\mathrm{p}}=R_{\text {eff }}$ and $3 R_{\text {eff }}$. The kinematic modelling by the observers ${ }^{4}$ yielded low mass-to-light ratios, e.g. $M / L \simeq 7$ at $5 R_{\text {eff }}$ for NGC 3379, consistent with a "naked" stellar population. They interpreted this as "little if any DM in these galaxies' haloes", in conflict with the conventional picture. While noticing that increasing velocity anisotropies could in principle produce declining $\sigma_{\mathrm{p}}$, they ruled out such "pathological" orbit structure. Similar conclusions were obtained later from other ellipticals. ${ }^{16}$ The apparent challenge to theory has already triggered radical explanations. ${ }^{17}$ However, the earlier analysis ${ }^{4}$ might have missed alternative solutions because it was limited to specific density-profile shapes in stationary spherical systems, the halo PNs were identified with the central stellar population, and their maximum-likelihood method may suffer from an incomplete orbit library or questionable convergence properties. ${ }^{18}$

\section{Anisotropic velocity dispersion}

For given density profiles, a lower $\sigma_{\mathrm{p}}$ can result from more radial velocities. The dynamics implies a lower 3D velocity dispersion $\sigma$ because the pressure needed for balancing gravity is provided by a radial $\sigma_{r}$ that corresponds to a lower $\sigma$. The projection introduces a further decrease in $\sigma_{\mathrm{p}}$. This can be illustrated by toy models made purely of circular or radial orbits, with the same constant speed and random orientations. If the stellar-density profile is steep enough, $\sigma_{\mathrm{p}}$ is dominated by the tangential contribution near the equatorial plane perpendicular to the line of sight, which is high for circular orbits and low for radial orbits.

The 3D profiles of any component of a spherical gravitating system in equilibrium obey the Jeans equation ${ }^{19}$ (derived from the collisionless Boltzmann equation),

$$
V^{2}(r)=[\alpha(r)+\gamma(r)-2 \beta(r)] \sigma_{r}^{2}(r)
$$

a manifestation of local hydrostatic balance between the inward pull of gravity (left) and the outward push of pressure (right). Here $V^{2}(r)=G M(r) / r$ is the squared circular velocity. 
The stellar density profile $\nu(r)$ enters via $\alpha \equiv-d \ln \nu / d \ln r$. Its velocity dispersion consists of radial and tangential components, $\sigma^{2}=\sigma_{r}^{2}+2 \sigma_{\theta}^{2}$; we define $\gamma \equiv-d \ln \sigma_{r}^{2} / d \ln r$. The velocity anisotropy is $\beta \equiv 1-\sigma_{\theta}^{2} / \sigma_{r}^{2}$, with $\beta=-\infty, 0,1$ for circular, isotropic and radial orbits respectively. The projection can be performed analytically when $\beta, \alpha$ and $\gamma$ are constant with $r$ (power-law profiles, $\left.V^{2}=V_{0}^{2}\left(r / R_{\text {eff }}\right)^{-\gamma}\right)$ :

$$
\sigma_{\mathrm{p}}^{2}\left(r_{\mathrm{p}}\right)=A(\alpha, \gamma)\left(\frac{(\alpha+\gamma)-(\alpha+\gamma-1) \beta}{(\alpha+\gamma)-2 \beta}\right) V_{0}^{2}\left(\frac{r_{\mathrm{p}}}{R_{\mathrm{eff}}}\right)^{-\gamma}
$$

( $A$ in Methods). Note that $\sigma_{\mathrm{p}}$ is a decreasing function of $\beta$ and of $\alpha$ (for $\alpha+\gamma>3$ and $\beta>0$ ) respectively. Local fits to the de-projection of the standard de Vaucouleurs ${ }^{20}$ surface-brightness profile of ellipticals give $\alpha \simeq 3.13-3.37$ at $2-3 R_{\text {eff. }}{ }^{21}$ Our fits to $\sigma_{\mathrm{p}}^{2}\left(r_{\mathrm{p}}\right)$ in the observed ellipticals beyond $R_{\text {eff }}$ yield $\gamma \simeq 0.8 \pm 0.2$. These give $A(\alpha, \gamma) \simeq 0.2$ and $\sigma_{\mathrm{p}}$ drops by a factor $\sim 1.5$ between $\beta=0$ and 1 . We learn that one could match the low $\sigma_{\mathrm{p}}$ at large radii either by a low $V_{0}$ or by a high $\beta$ there, and that a high $\alpha$ helps.

In a more realistic model, we assume a Sérsic stellar density profile ${ }^{22,23,21}$ (= de Vaucouleurs' for Sérsic index $m=4)$, a standard DM density profile ${ }^{7}$ with a typical concentration $(\sim 10),{ }^{24}$ and a virial stellar mass fraction $\sim 0.06$. With $\beta=0$ we recover the apparent 5D discrepancy, but $m \simeq 4$ and $\beta\left(r>R_{\text {eff }}\right) \simeq 0.5$ (independent of its behaviour well inside $R_{\text {eff }}$ ) yield a $\sim 1 \sigma$ agreement with the observed $\sigma_{\mathrm{p}}$. A good fit is obtained with either $m \simeq 4$ and $\beta \simeq 0.75$, or with $m \simeq 2.3$ (for which $\alpha \simeq 3.5$ near $2.5 R_{\text {eff }}$ ) and $\beta \simeq 0.5$. The required $\beta$ is higher than the $\beta \leq 0.2$ predicted for DM particles in typical haloes at a few $R_{\text {eff }}\left(\ll R_{\text {vir }}\right){ }^{15}$ so the PNs must not trace the DM kinematics.

\section{Merger simulations}

Assuming that ellipticals form by mergers, and that major mergers of discs can reveal generic features of mergers in the $\Lambda$ CDM cosmology, we appeal to a suite of simulations of such events. ${ }^{5,6}$ Two spirals are put on a parabolic orbit, each consisting of stellar and gaseous discs and a bulge, all embedded in a $\Lambda$ CDM halo, constructed to match a range of typical disc galaxies. The gravitational and hydrodynamical evolution is followed using an SPH code, ${ }^{25}$ including gas cooling, star formation and supernova feedback (Methods).

Fig. 1 shows the stacked 3D profiles of the merger remnants. The DM density profile is slightly flatter than an isothermal sphere, $\rho \propto r^{-2}$, similar to simulated $\Lambda$ CDM haloes after they have responded to gas dissipation. ${ }^{26}$ The robust stellar density falls off more steeply, $\rho \propto r^{-3.2}$, as in elliptical galaxies obeying the de Vaucouleurs profile near 2-3 $R_{\text {eff }}$, and with $R_{\text {eff }} \simeq 0.015 R_{\text {vir }}$. For the "young" stars it is somewhat steeper, $\rho \propto r^{-3.5}$. The total-to-stellar mass ratio rises from $\simeq 2$ at $3 R_{\text {eff }}$ to $\simeq 14$ at $R_{\text {vir }}$, corresponding at $5 R_{\text {eff }}$ to $M / L \simeq 15$ (compared to the earlier ${ }^{4} M / L \simeq 7$ ) (both for stellar $M_{*} / L=6$ ). 5D The $3 \mathrm{D} \sigma$ profiles of the DM and stars have similar slopes [as in eq. (2)], falling off roughly as $\sigma \propto r^{-0.2}$.

Our main new finding is the high $\beta$ of the stellar halo velocities. While the DM velocities are almost isotropic $(\beta \sim 0.1)$, the typical stellar $\beta$ grows from small values at $r<R_{\text {eff }}$ 

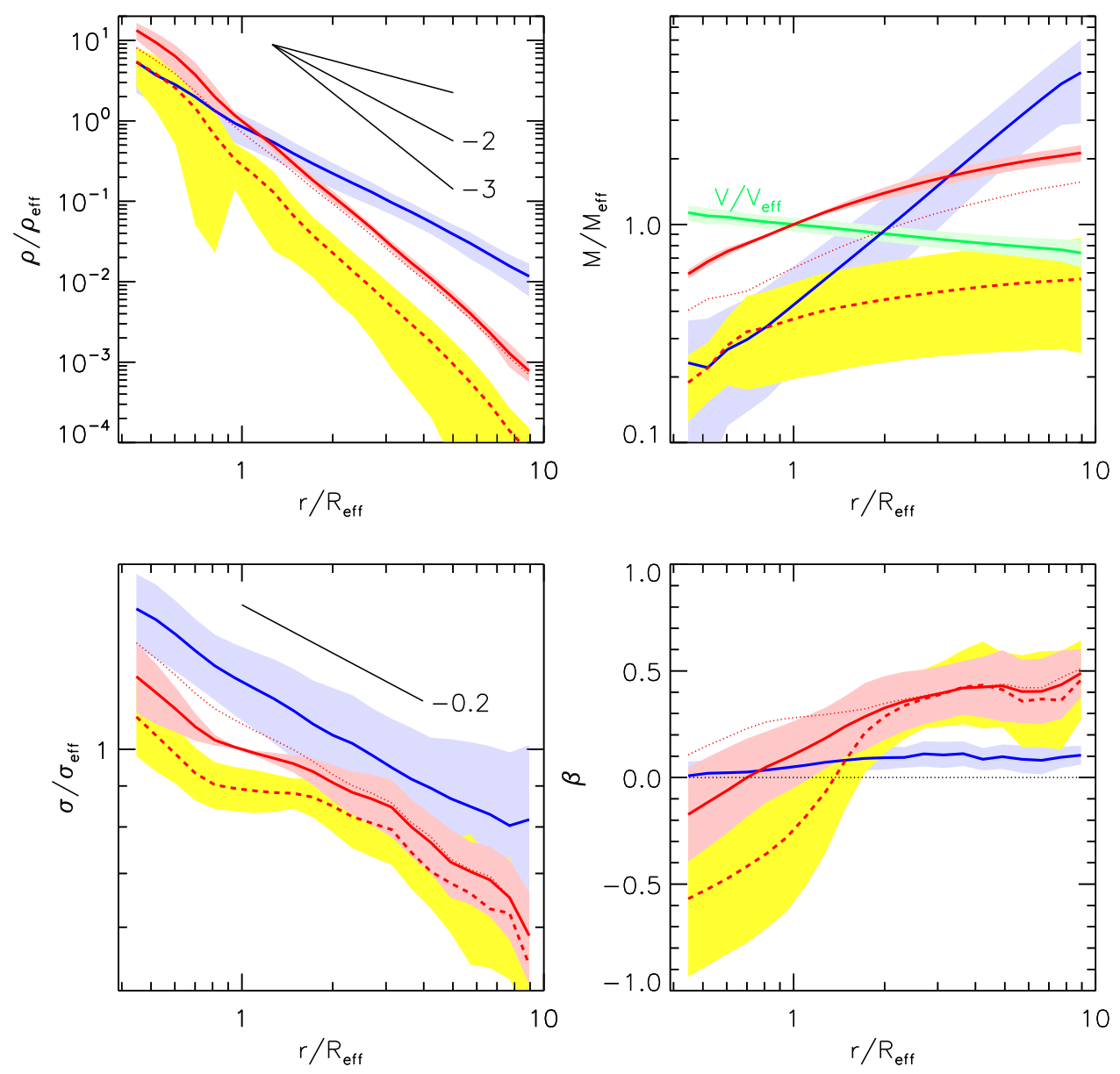

Figure 1. Three-dimensional profiles of the simulated merger remnants. Ten galaxies at two different times after the merger (typically 0.8 and $1.3 \mathrm{Gyr}$ ) are stacked together. Shown are the profiles for the dark matter (blue) and the stars (red), divided into the old ones from the progenitors (dotted) and those newly formed during the merger (dashed). The scaling is such that the curves for the stars (all, solid red) are matched at $R_{\text {eff. }}$ The shaded areas mark $1 \sigma$ scatter. The panels refer to density $\rho$, mass $M$ and circular velocity $V$, velocity dispersion $\sigma$ and anisotropy $\beta$, with "eff" referring to the quantities at $R_{\text {eff }}$. 
(sometimes negative, reflecting a small disc that is irrelevant to the profiles beyond $R_{\text {eff }}$ ) to $\beta \sim 0.5$ at $r>R_{\text {eff. }}$. In one case $\beta>0.75$, but in another it remains $<0.2$. Given $V(r)$ in the Jeans equation, the higher $\alpha$ is compensated by a higher $\beta$ and a lower $\sigma$.

The simulations demonstrate that the stellar halo originates from tidal processes during the first pericentre passage. Some of the halo stars are associated with the two cores and the tidal bridge between them; they pass near the center at the coalesence before flying outward on radial orbits. Other halo stars first flow out in extended tidal tails and later fall back on radial orbits [Supplementary Information, hereafter SI]. Indeed, we find that $\beta$ is correlated with the strength of the tidal interaction; it is higher for more head-on collisions and when the spins are aligned with the orbit.

\section{Projected profiles}

The systems are "observed" from three orthogonal directions and stacked together, providing a robust average profile and the scatter about it. The data are scaled similarly (Methods). Fig. 2 shows the simulated surface-density profile and those of NGC 821, ${ }^{27}$ $3379^{28}$ and $4697 .{ }^{29}$ They are all fit by $\Sigma \propto r_{\mathrm{p}}^{-2.3}$ in the range $1-5 R_{\text {eff }}$ (as in de Vaucouleurs profile at $\left.\sim 2 R_{\text {eff }}\right)$. The simulated projected axial ratios near $R_{\text {eff }}$ range from 1:1 to 1:2, and the ellipticity is supported by an anisotropic, triaxial velocity dispersion rather than by rotation, similar to ellipticals [SI]. In addition, The distribution of global properties of the simulated remnants, such as luminosity, radius and velocity dispersion, is consistent with the "fundamental plane" of ellipticals [SI]. Thus, the merger remnants seem to resemble typical ellipticals near $\sim R_{\text {eff }}$ in every relevant respect.

The velocity dispersions in Fig. 2 demonstrate our punchline. While the DM $\sigma_{\mathrm{p}}$ indeed lies above the outer observed points, the stellar $\sigma_{\mathrm{p}}, \sim 30 \%$ lower, provides a good fit everywhere in the range $0.5-4 R_{\text {eff }}$. The slope of the simulated $\sigma_{\mathrm{p}}^{2}\left(r_{\mathrm{p}}\right)$ in the range $0.5-6 R_{\text {eff }}$ is $\gamma=0.53 \pm 0.16$ for "all" and $\gamma=0.61 \pm 0.22$ for the "young" stars, both consistent with the $\gamma=0.59 \pm 0.13$ observed for PNs. No fiddling with model parameters is involved simply stacking a sample of merger remnants as simulated.

The $\sigma_{\mathrm{p}}$ of "young" stars is lower by $\sim 9 \%$ at $3 R_{\text {eff }}$ (due to their larger $\alpha$ ). Stellar theory indicates that these objects, $<3 \mathrm{Gyr}$ old, may represent the observed PNs. Emerging from 1.4-2.5 $M_{\odot}$ stars, ${ }^{30}$ the PNs are expected to be much more luminous than those of the older, less massive stars, which fall below the detection limits ${ }^{3,4}$ (Methods). However, the radial orbits are a generic result independent of the degree of gas dissipation during the merger: our mergers with initial gas-to-baryon ratio ranging from 0 to $70 \%$ show negligible differences in $\beta$ beyond $R_{\text {eff }}$. Dissipation results in a more centrally concentrated stellar distribution, associated with $\lesssim 10 \%$ reduction in outer $\sigma_{\mathrm{p}}$ [SI]. We also find that the radial orbits and low $\sigma_{\mathrm{p}}$ emerge from major and minor mergers alike, independent of the progenitor mass ratio [SI], and that the presence of a $\sim 22 \%$ bulge does not make a significant difference. The tidal origin of the stellar halo explains this robustness to many merger characteristics. Furthermore, one simulation continued till 3.5 Gyr after the merger with no sign of evolution in $\beta(r)$ and $\sigma_{\mathrm{p}}(r)$ beyond $\sim R_{\text {eff }}[\mathrm{SI}]$. 

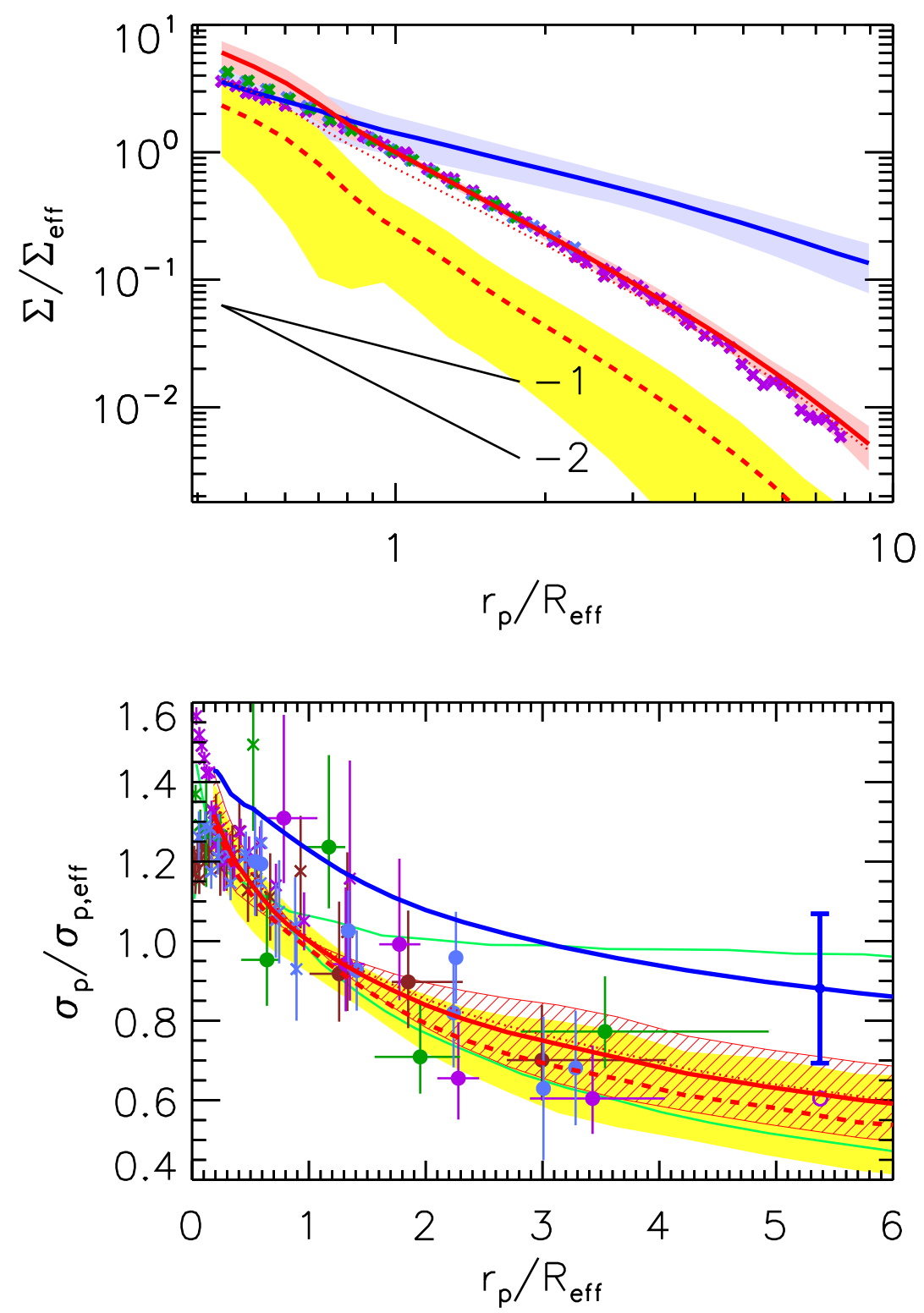

Figure 2. Projected profiles: simulated galaxies versus observations. Top: surface density. Bottom: velocity dispersion. The merger remnants are viewed from three orthogonal directions and the 60 profiles are stacked such that the stellar curves ("all") match at $R_{\text {eff }}$. Colors and line types are as in Fig. 1. The $<3$ Gyr "young" stars may mimic the observed PNs. The $1 \sigma$ scatter is marked by shaded or hashed areas or a thick bar. The galaxies are marked green (821), violet (3379), brown (4494) and blue (4697) with $1 \sigma$ errors; PNs (circles) and stars (crosses). The surface densities shown for 3 galaxies almost coincide with the simulated profile. Green lines refer to the earlier models ${ }^{4}$ with (upper) and without (lower) DM. 
The $\pm 20 \%$ scatter in $\sigma_{\mathrm{p}}$ is partly due to the angular momentum of the merger orbit and the relative spin inclinations, but also due to the line-of-sight relative to the principal axes of the triaxial velocity-dispersion tensor (or rotation axis, A. Burkert et al., in preparation). When viewed "face-on", some of the remnants show $\sigma_{\mathrm{p}}$ lower than observed, while other extreme "edge-on" cases show $\sigma_{\mathrm{p}}$ almost as high as that of the DM [SI].

The simulated line-of-sight velocity distribution (LOSVD) is consistent with the data also beyond the second moment. Both the simulated and observed deviations from a Gaussian distribution are typically small, with the fourth moment $h_{4}=0.03 \pm 0.05$ for the central stars [SI]. At $r \gtrsim R_{\text {eff }}$, radial, prograde mergers produce negligible $h_{4}$ values as in the PNs of NGC $3379^{4}$ (or small positive values as in NGC 5128 ${ }^{31}$ ), while more circular and retrograde mergers, or gas-rich mergers, can produce negative $h_{4}$ as in NGC $4697^{3}$ [SI].

\section{Conclusion}

We conclude that the PN data are consistent with the simple picture where normal ellipticals also live in massive DM haloes. The low $\sigma_{\mathrm{p}}$ is primarily due to the radial orbits of the halo stars, being tidally ejected from the inner regions during mergers independently of dissipation and mass ratio. This generic origin of the radial orbits indicates that the results based on our sample of simulations are representative of a broader range of merger types expected in the $\Lambda \mathrm{CDM}$ cosmology, and argues that the low observed PN velocities are a natural outcome of this standard model. This should be confirmed by cosmological simulations (e.g. low-resolution results, ${ }^{32}$ and work in preparation by J. Navarro et al.).

The range of merger properties leads to a variety of $\beta$ and $\sigma_{\mathrm{p}}$ profiles, and the triaxiality adds directional variations, allowing extreme $\sigma_{\mathrm{p}}$ values smaller and larger than the PN data. The possible association of the PNs with the younger stars, whose density profile is slightly steeper, may help reducing their $\sigma_{\mathrm{p}}$ a bit further. Other tracers, involving old stars, are expected to show a somewhat higher $\sigma_{\mathrm{p}}$. This is especially true for globular clusters, ${ }^{33,16}$ given their flatter density profile ${ }^{34}$ and presumably lower anisotropy (due to tidal disruption in radial orbits). A somewhat higher $\sigma_{\mathrm{p}}$ may also be expected in gas-poor elliptical-elliptical mergers, common especially in groups (as observed ${ }^{16}$ ), where the collision orbits may be circularized by two-body relaxation and dynamical friction.

Recall ${ }^{4}$ that a nearly isotropic "naked" stellar system also provides a fit to the $\sigma_{\mathrm{p}}\left(r_{\mathrm{p}}\right)$ data inside a few $R_{\text {eff }}$, appealing to a low $V_{0}$ rather than a high $\beta$ in eq. (2). Our simulations provide another solution, which does include a standard DM halo. The DM model predicts that $\sigma_{\mathrm{p}}$ flattens toward $\sim 10 R_{\text {eff }}$ (as in NGC 5128 ${ }^{31}$ ), where the "naked" model predicts a continuing decline beyond $3 R_{\text {eff }}$ and very low $\sigma_{\mathrm{p}}$ for other tracers as well. While the "naked" model violates much of what we know about galaxy formation and cosmology, the DM model, with the radial stellar orbits, seems to be a straightforward outcome of the self-consistent picture of structure formation in the Universe. 


\section{Methods}

\section{Equation 2}

The coefficient in eq. (2) is

$$
A(\alpha, \gamma)=\frac{1}{(\alpha+\gamma)} \frac{\Gamma[(\alpha+\gamma-1) / 2]}{\Gamma[(\alpha+\gamma) / 2]} \frac{\Gamma[\alpha / 2]}{\Gamma[(\alpha-1) / 2]} .
$$

This is a weak function of $\alpha$ and $\gamma$. For instance: $A(3.5,1.0) \simeq 0.18, A(3.0,1.0) \simeq 0.20$, $A(3.5,0.4) \simeq 0.23, A(3.0,0.4) \simeq 0.26$.

\section{Merger simulations}

The merger simulations ${ }^{5,6}$ represent some of the major collisions that likely occurred during the hierarchical structure formation according to the $\Lambda$ CDM cosmology. The evolution is followed using the entropy-conserving, gravitating, Smoothed Particle Hydrodynamics (SPH) code GADGET. ${ }^{25}$ Gas cooling, star formation and supernova feedback are treated using simplified recipes that were calibrated to match observed star-formation rates.

The progenitor disc galaxies mimic typical big spirals galaxies: one type $(\mathrm{G})$ representing today's Sb galaxies and another type containing more gas as in Sbc-Sc galaxies and at high redshift. The sample consists of four G mergers, with DM masses $1.2 \times 10^{12} M_{\odot}$ (except one $\left.5 \times 10^{11} M_{\odot}\right)$, and five Sbc mergers plus one Sc merger with $8 \times 10^{11} M_{\odot}$ haloes. The baryonic fraction is $\sim 5 \%$ of the DM halo mass in the $\mathrm{G}$ cases, and $\sim 13 \%$ in the Sbc-Sc cases. The fraction of baryons in gas is $\sim 20 \%$ in G, $52 \%$ in Sbc, and $70 \%$ in Sc. The particle mass is $<10^{6} M_{\odot}$ for gas and stars and $\lesssim 10^{7} M_{\odot}$ for DM. The smoothing lengths are $h=100$ and 400 pc with the force becoming Newtonian at $\geq 2.3 h$.

Two identical galaxies are set on parabolic orbits and merge because of dynamical friction due to their massive haloes. Our sample consists of several different orbits and orientations, including prograde and retrograde configurations in which the galaxy spins are aligned or antialigned with the collision orbital angular momentum. The merger results in two succesive starbursts, one after the first close approach, and the other after the second, final coalescence [SI]. The starbursts typically occur 1-2 Gyr after the beginning of the simulation, and the remnant is "observed" $\sim 1$ Gyr later. The amount of stars formed during the merger is roughly proportional to the initial gas fraction, and is not too sensitive to the orbit or orientation. The instantaneous rate varies in the range 10-100 $M_{\odot} / \mathrm{yr}$. The young stars formed during the merger constitute $\sim 30 \%$ of the total stars; typically $20 \%$ in the $\mathrm{G}$ cases and $40 \%$ in Sbc. The remnant galaxies resemble normal elliptical galaxies, as demonstrated above [SI].

The smaple used from the suite of 1:1 merger simulations ${ }^{5,6}$ consists of the following runs:

The $G$ cases are: G3G3b-u1 (the fiducial G case, a gas-poor galaxy obeying the scaling relations from SDSS), G3blv5G3blv5b-u1 (bulgeless), G3G3r-u1 (a retrograde orbit), G2G2r-u1 (a slightly smaller gas-poor galaxy obeying the SDSS scalings, on a retrograde orbit).

The Sbc-Sc cases are: Sbc201a-u4 (the fiducial Sbc case, gas rich, small bulge, same as run n2low ${ }^{6}$ ), Sbc201-uu43 (with stronger feedback, n2high ${ }^{6}$ ), Sbc203-u4 (retrograde orbit), Sbc204-u4 (very radial orbit), Sbc205-u4 (more tangential orbit), Sc201-u4 (mimicking Sc galaxies with no bulge and a higher gas fraction). 


\section{Scaling the data}

The observed galaxies are presented together using the $R_{\text {eff }}$ determined from each surfacebrightness profile. ${ }^{28,29,4}$ We note that $R_{\text {eff }}$ for NGC $3379^{28,29}$ is $\simeq 50 \%$ larger than that quoted. ${ }^{4}$ In Fig. 2, an open circle marks the last point had we used the smaller ${ }^{4} R_{\text {eff }}$; this uncertainty does not make a qualitative difference. The amplitudes of $\Sigma$ and $\sigma_{\mathrm{p}}$ are scaled by least-squares fits of the stellar data at $r>0.2 R_{\text {eff }}$ to the stacked simulated profile as a reference. Replacing this reference by a different function of a similar general shape yields similar results. Using only the stars at larger radii (up to $r>R_{\text {eff }}$ ), or using the PNs alone, yield $\sigma_{\mathrm{p}}$ adjusting factors that differ only by a few percent.

The $R_{\text {eff }}$ of NGC 821, 3379, 4494, 4697 match the mean simulation value after multiplication by $0.667,1.57,1.00,1.13$, indicating that the simulated and observed galaxies are of comparable sizes. The $\sigma_{\mathrm{p}}$ were adjusted by factors $1.00,1.19,1.21,1.11$ for best fit. Being comparable to the radius scaling factors indicates that the observed and simulated galaxies have a similar velocity structure. The mean and $1 \sigma$ scatter in the simulated remnants are $R_{\text {eff }}=4.05 \pm 1.04 \mathrm{kpc}$ and $\sigma_{\mathrm{p}}\left(R_{\mathrm{eff}}\right)=154 \pm 33 \mathrm{~km} \mathrm{~s}^{-1}$.

\section{Age of Planetary Nebulae}

The [OIII] luminosity of a PN with mass $<2.5 M_{\odot}$ is strongly increasing with the parent stellar mass $^{30}$ [Figs. 10, 14], hence sharply decreasing with age. A limiting magnitude $M_{5007}$ then corresponds to a maximum stellar age $t$. For the complete sample of $328 \mathrm{PNs}$ in NGC $4697^{3}$ it is $M_{5007} \simeq-2.6$, namely $t_{M 01}<3 \mathrm{Gyr}^{30}$ [Figs. 18, 19, 26]. With only $\simeq 100 \mathrm{PNs}$ per galaxy, ${ }^{4}$ the magnitude limit is brighter (by -0.8 magnitudes based on telescope gathering areas), so the stars are even younger. Based on theoretical PN luminosity functions ${ }^{30}$ [Figs. 18, 26], if the population is typically older than 1 Gyr then $t_{R 03}<2$ Gyr. We therefore adopt $t<3$ Gyr as a limit for most of the observed PNs in the four galaxies. This indicates an association with the "young" simulated stars, and that the mergers of gaseous discs are relevant to those ellipticals showing PNs. A caveat is the apparent relative invariance of the PN luminosity function between galaxies, seemingly independent of signs for a recent major merger. When there are no signs for a recent major merger, the observed PNs, if indeed young, may be the signature of recent minor mergers, which are expected to produce similar effects. 
Received; Accepted .

1. Sofue, Y. \& Rubin, V. Rotation Curves of Spiral Galaxies. ARA\&A 39, 137-174 (2001).

2. White, S. D. M. \& Rees, M. J. Core condensation in heavy halos - A two-stage theory for galaxy formation and clustering. MNRAS 183, 341-358 (1978).

3. Méndez, R. H. et al. Detection, Photometry, and Slitless Radial Velocities of 535 Planetary Nebulae in the Flattened Elliptical Galaxy NGC 4697. ApJ 563, 135-150 (2001).

4. Romanowsky, A. J. et al. A Dearth of Dark Matter in Ordinary Elliptical Galaxies. Science 301, 1696-1698 (2003).

5. Cox, T. J. Simulations of Galaxy Mergers: Star Formation and Feedback. PhD thesis UC Santa Cruz, supervisor: J.R. Primack (2004). http://physics.ucsc.edu/ tj/work/thesis/.

6. Cox, T. J., Jonsson, P., Primack, J. R. \& Somerville, R. S. The Effects of Feedback in Simulations of Disk Galaxy Major Mergers. astro-ph/0503201 (2005).

7. Navarro, J. F., Frenk, C. S. \& White, S. D. M. A Universal Density Profile from Hierarchical Clustering. ApJ 490, 493-510 (1997).

8. Prada, F. et al. Observing the Dark Matter Density Profile of Isolated Galaxies. ApJ 598, 260-271 (2003).

9. Blumenthal, G. R., Faber, S. M., Primack, J. R. \& Rees, M. J. Formation of galaxies and large-scale structure with cold dark matter. Nature 311, 517-525 (1984).

10. Dekel, A. \& Silk, J. The origin of dwarf galaxies, cold dark matter, and biased galaxy formation. ApJ 303, 39-55 (1986).

11. Fall, S. M. Dissipation, merging and the rotation of galaxies. Nature 281, 200-202 (1979).

12. Mathews, W. G. \& Brighenti, F. Hot Gas in and around Elliptical Galaxies. ARA\&A 41, 191-239 (2003).

13. Keeton, C. R. Cold Dark Matter and Strong Gravitational Lensing: Concord or Conflict? ApJ 561, 46-60 (2001).

14. Mateo, M. L. Dwarf Galaxies of the Local Group. ARA\&A 36, 435-506 (1998).

15. Mamon, G. A. \& Lokas, E. L. Confronting LCDM with the optical observations of elliptical galaxies: II. Weighing the dark matter component. astro-ph/0405491 (2005).

16. Napolitano, N. R., Capaccioli, M., Romanowsky, A. J., Douglas, N. G., Merrifield, M. R., Kuijken, K., Arnaboldi, M., Gerhard, O. \& Freeman, K. C. Mass-to-light ratio gradients in early-type galaxy haloes. MNRAS 357, 691-706 February 2005.

17. Milgrom, M. \& Sanders, R. H. Modified Newtonian Dynamics and the "Dearth of Dark Matter in Ordinary Elliptical Galaxies". ApJ 599, L25-L28 (2003). 
18. Valluri, M., Merritt, D. \& Emsellem, E. Difficulties with Recovering the Masses of Supermassive Black Holes from Stellar Kinematical Data. ApJ 602, 66-92 February 2004.

19. Binney, J. \& Tremaine, S. Galactic dynamics. Princeton, NJ, Princeton University Press (1987).

20. de Vaucouleurs, G. Recherches sur les Nebuleuses Extragalactiques. Annales d'Astrophysique 11, 247-287 (1948).

21. Mamon, G. A. \& Lokas, E. L. Confronting LCDM with the optical observations of elliptical galaxies: I. Is the total mass density profile of the NFW form or even steeper? astroph/0405466 (2005).

22. Sersic, J. L. Atlas de galaxias australes. Cordoba, Argentina: Observatorio Astronomico (1968).

23. Caon, N., Capaccioli, M. \& D'Onofrio, M. On the Shape of the Light Profiles of Early Type Galaxies. MNRAS 265, 1013-1021 (1993).

24. Bullock, J. S. et al. Profiles of dark haloes: evolution, scatter and environment. MNRAS 321, 559-575 (2001).

25. Springel, V., Yoshida, N. \& White, S. D. M. GADGET: a code for collisionless and gasdynamical cosmological simulations. New Astronomy 6, 79-117 (2001).

26. Gnedin, O. Y., Kravtsov, A. V., Klypin, A. A. \& Nagai, D. Response of Dark Matter Halos to Condensation of Baryons: Cosmological Simulations and Improved Adiabatic Contraction Model. ApJ 616, 16-26 (2004).

27. Goudfrooij, P., Hansen, L., Jorgensen, H. E., Norgaard-Nielsen, H. U., de Jong, T. \& van den Hoek, L. B. Interstellar matter in Shapley-Ames elliptical galaxies. I. Multicolour CCD surface photometry. A\&AS 104, 179-231 April 1994.

28. de Vaucouleurs, G. \& Capaccioli, M. Luminosity distribution in galaxies. I - The elliptical galaxy NGC 3379 as a luminosity distribution standard. ApJS 40, 699-731 (1979).

29. Peletier, R. F., Davies, R. L., Illingworth, G. D., Davis, L. E. \& Cawson, M. CCD surface photometry of galaxies with dynamical data. II - UBR photometry of 39 elliptical galaxies. AJ 100, 1091-1142 (1990).

30. Marigo, P., Girardi, L., Weiss, A., Groenewegen, M. A. T. \& Chiosi, C. Evolution of planetary nebulae. II. Population effects on the bright cut-off of the PNLF. A\&A 423, 995-1015 (2004).

31. Peng, E. W., Ford, H. C. \& Freeman, K. C. The Planetary Nebula System and Dynamics in the Outer Halo of NGC 5128. ApJ 602, 685-704 (2004).

32. Sáiz, A., Domínguez-Tenreiro, R. \& Serna, A. Elliptical Galaxies at z=0 from Self-consistent Hydrodynamical Simulations: Comparison with Sloan Digital Sky Survey Structural and Kinematical Data. ApJ 601, L131-L134 (2004).

33. Zepf, S. E., Bergond, G., Romanowsky, A. J., Rhode, K. L. \& Sharples, R. M. Some Dynamical Constraints on the Halo of NGC 3379 from Wide-Field Spectroscopy of Its Globular Cluster System. American Astronomical Society Meeting Abstracts 205, 3104-3104 (2004). 
34. Côté, P. et al. Dynamics of the Globular Cluster System Associated with M87 (NGC 4486). II. Analysis. ApJ 559, 828-850 (2001).

35. Khochfar, S. \& Burkert, A. Orbital Parameters of Merging Dark Matter Halos astro$\mathrm{ph} / 0309611$ (2003).

36. Helsdon, S. F. \& Ponman, T. J. The morphology-density relation in X-ray-bright galaxy groups. MNRAS 339, L29-L32 March 2003.

37. Bendo, G. J. \& Barnes, J. E. The line-of-sight velocity distributions of simulated merger remnants. MNRAS 316, 315-325 August 2000.

38. Cretton, N., Naab, T., Rix, H. \& Burkert, A. The Kinematics of 3:1 Merger Remnants and the Formation of Low-Luminosity Elliptical Galaxies. ApJ 554, 291-297 June 2001.

39. Bender, R., Saglia, R. P. \& Gerhard, O. E. Line-of-Sight Velocity Distributions of Elliptical Galaxies. MNRAS 269, 785-+ August 1994.

40. Statler, T. S. \& Smecker-Hane, T. The Stellar Kinematic Fields of NGC 3379. AJ 117, 839-854 February 1999.

41. Pinkney, J., Gebhardt, K., Bender, R., Bower, G., Dressler, A., Faber, S. M. \& Filippenko, A. V. et al. Kinematics of 10 Early-Type Galaxies from Hubble Space Telescope and Groundbased Spectroscopy. ApJ 596, 903-929 October 2003.

42. Emsellem, E., Cappellari, M., Peletier, R. F., McDermid, R. M., Bacon, R., Bureau, M. \& Copin, Y. et al. The SAURON project - III. Integral-field absorption-line kinematics of 48 elliptical and lenticular galaxies. MNRAS 352, 721-743 August 2004.

43. Gerhard, O. E. Line-of-sight velocity profiles in spherical galaxies: breaking the degeneracy between anisotropy and mass. MNRAS 265, 213-+ November 1993.

44. Rothberg, B. \& Joseph, R. D. A Deep K-Band Photometric Survey of Merger Remnants. AJ 128, 2098-2143 November 2004.

45. Veilleux, S., Kim, D.-C. \& Sanders, D. B. Optical and Near-Infrared Imaging of the IRAS 1 Jy Sample of Ultraluminous Infrared Galaxies. II. The Analysis. ApJS 143, 315-376 December 2002 .

46. de Zeeuw, T. \& Franx, M. Structure and dynamics of elliptical galaxies. ARA\&A 29, 239-274 (1991).

47. Ryden, B. The intrinsic shapes of elliptical galaxies. ApJ 396, 445-452 September 1992.

48. Alam, S. M. K. \& Ryden, B. S. The Shapes of Galaxies in the Sloan Digital Sky Survey. ApJ 570, 610-617 May 2002.

49. Statler, T. S. \& SAURON Team NGC 4365: A Truly Triaxial Galaxy. AAS/Division of Dynamical Astronomy Meeting 34,-+ August 2003.

50. Naab, T. \& Burkert, A. Statistical Properties of Collisionless Equal- and Unequal-Mass Merger Remnants of Disk Galaxies. ApJ 597, 893-906 (2003). 
51. Springel, V. \& Hernquist, L. Formation of a Spiral Galaxy in a Major Merger. ApJ 622, L9-L12 March 2005.

52. González-García, A. C. \& Balcells, M. Elliptical galaxies from mergers of discs. MNRAS 357, 753-772 February 2005.

53. Ciardullo, R., Sigurdsson, S., Feldmeier, J. J. \& Jacoby, J. H. Close Binaries as the Progenitors of the Brightest Planetary Nebulae. astro-ph/0504619 (2005).

54. Cretton, N. \& Emsellem, E. On the reliability of the black hole mass and mass-to-light ratio determinations with Schwarzschild models. MNRAS 347, L31-L35 January 2004.

55. Richstone, D. et al. Black Hole Mass Determinations From Orbit Superposition Models are Reliable. astro-ph/0403257 (2004).

Acknowledgments We acknowledge stimulating discussions with M. Beasley, A. Burkert, K. Gebhardt, J. Navarro, A. Romanowsky and his group, ${ }^{4}$ and assistance from M. Covington. This research has been supported by ISF 213/02 and by NASA ATP NAG5-8218. AD acknowledges a Miller Professorship at UC Berkeley, support from UCO/Lick Observatory, and a Blaise Pascal International Chair in Paris.

Competing interests statement The authors declare that they have no competing financial interests.

Correspondence and requests for materials should be addressed to A.D. (dekel@phys.huji.ac.il). 


\section{SUPPLEMENTARY INFORMATION}

The following is an extension of the Letter to Nature, aimed at providing more detailed support to the results reported in the Letter.

\section{Outline:}

1. The generic origin of a stellar halo with radial orbits

2. Robustness to dissipation

3. Robustness to major versus minor mergers

4. Robustness to the presence of a bulge

5. Long-term stability

6. Parametrizing the velocity-dispersion profiles

7. The full LOSVD and the $h_{4}$ moment

8. Matching other properties of ellipticals

9. Scatter induced by triaxiality

10. On the PN age

11. Rising versus constant $\beta$

12. Why was the DM solution missed before?

13. Robustness and Uniqueness of the results

Fig. 3 shows snapshots from one of our merger simulations, brought here for general impression of some of the issues discussed in the Letter and in this SI.

\section{The generic origin of a stellar halo with radial orbits}

Fig. 4 demonstrates the important role of tidal processes in the formation of a stellar halo and its radial orbits. The final halo stars are composed of two populations: (a) stars that passed through the center near the second-pericentre coalescence, some of which were part of the tidal bridge formed after the first-pericentre passage, and (b) stars that first flew outward in extended tidal tails after the first-pericentre passage and then fell back after the merger of the cores.

The tidal origin of the radial orbits explains the robustness to the degree of dissipation, the progenitor mass ratio, the bulge fraction and so on. It predicts that $\beta$ should predominantly be a function of the strength of the tidal interactions associated with the collision. Indeed, we find in our simulations a strong tendency for high $\beta$ in collisions of low impact parameter, as well as in prograde collisions, where the tidal effects are stronger. High angular momentum collisions, and retrograde collisions, typically lead to low beta values. Such collisions may be responsible for 


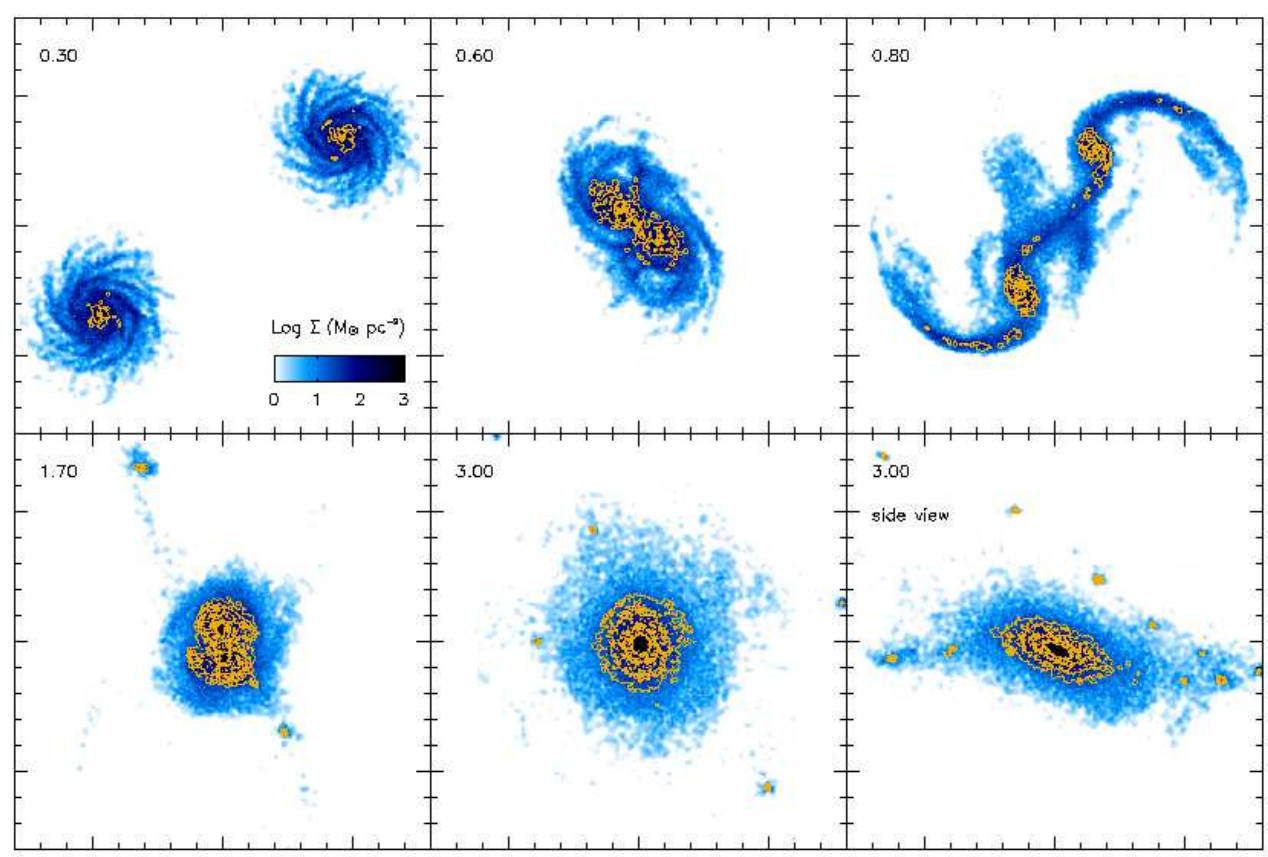

Figure 3. Visual impression. Stellar density maps in one of the merger simulations (fiducial Sbc). "All" the stars (blue) and the "young" stars formed during the merger (yellow contours). The time sequence (time marked in Gyr) is viewed "face-on" in the plane defined by one of the initial discs and the two centers of mass. Young stars form especially after each of the two pericentres, at $\sim 0.60$ and $\sim 1.70$ Gyr after the beginning of the simulation. The final system is also viewed "edge-on" (bottom-right), showing an axial ratio of roughly 1:2. The "young" stars have a somewhat steeper density profile and a slightly higher ellipticity.

elliptical galaxies with higher velocity dispersions than the ones discussed in the Letter. Future work may try to understand how such collisions are associated with bigger ellipticals preferentially in group centers (e.g. due to two-body relaxation and orbit circularization by dynamical friction).

How sensitive are our results to the choice of parabolic orbits in the simulations? We extracted our typical orbits from cosmological simulations. ${ }^{35}$ We have performed a few tests of different orbits and orientations, but have not obtained conclusive results about the trends. What we can say at this point is that the sample of simulations that we have used spans a range of properties that matches many of the real galaxy properties, and especially reproduces many cases of low $\sigma_{\mathrm{p}}$. The dependence on orbit is worth further study.

\section{Robustness to dissipation}

We find that the role of dissipation on the orbit anisotropy beyond $R_{\text {eff }}$ is negligible. The main result of dissipation is a more centrally concentrated stellar distribution, which results in $\sim 10 \%$ reduction in velocity dispersion - a minor effect. 


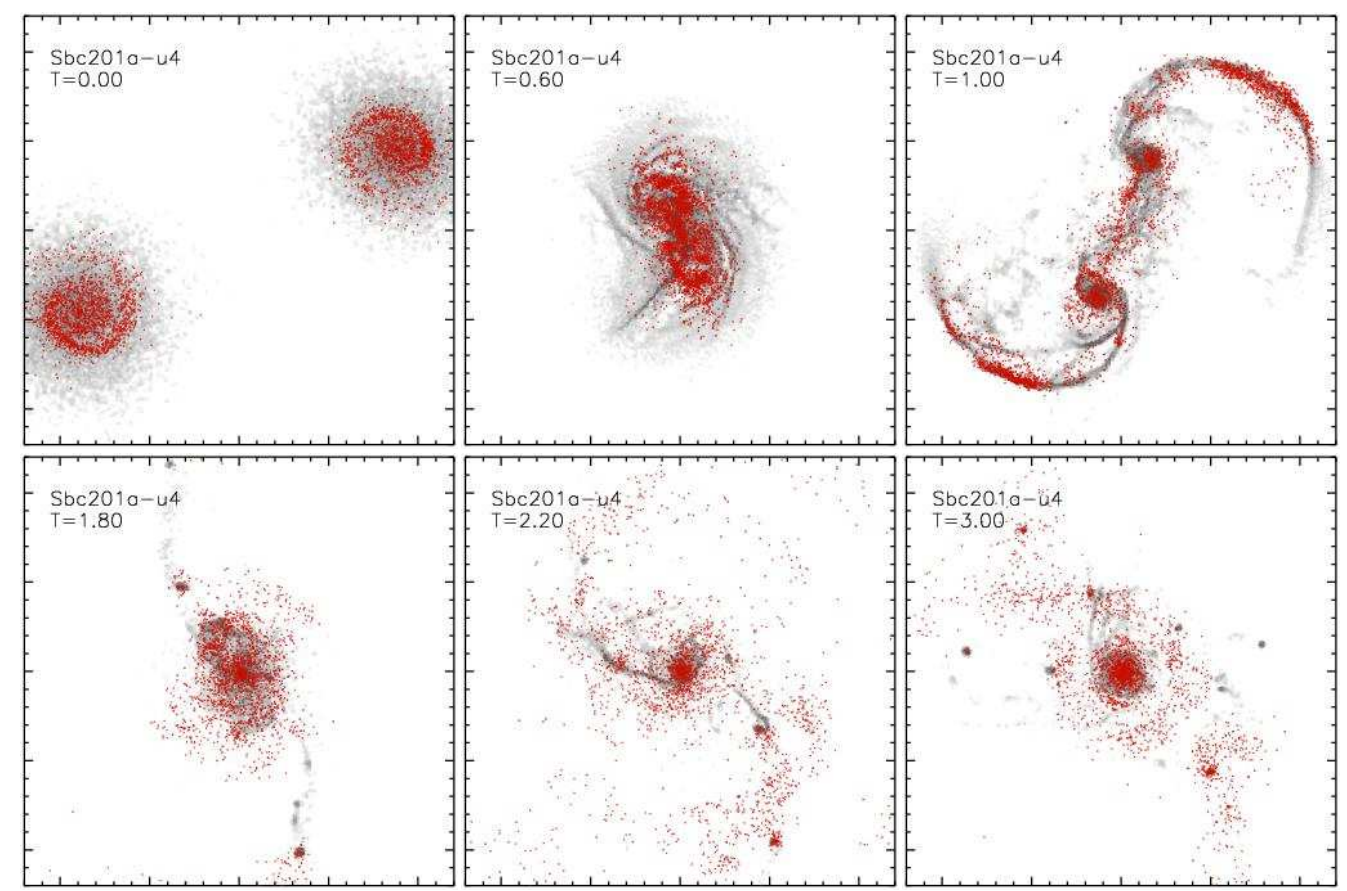

Figure 4. The tidal origin of the stellar halo and radial orbits is demonstrated via Snapshots from the fiducial Sbc simulation (same as Fig. 3). Marked red is a sample of ("old") stars identified at the final time $(t=3)$ as loosely bound. These stars are shown in their positions at several stages along the merger process. The final $R_{\text {eff }}$ is about two small tick marks. At the initial time, these stars are spread throughout the progenitor disks $(t=0)$. The first pericentre passage is at $t \simeq 0.6$, and during the rebound $(t=1.0)$ these stars are divided into two groups: those that are flying outward with the extended tidal tails, and those that are still associated with the progenitor cores as well as the tidal bridge between them. At the second-pericentre coalescence $(t \simeq 1.8)$, the outer tidal tails are not seen while the inner population is expanding from the center into the halo on highly radial orbits. At $t=2.2$ we see the stars from the tidal tails falling back in on highly radial orbits, to eventually join the inner group in a loosely bound stellar halo extending to a few $R_{\text {eff }}$.

One demonstration is provided by the comparison of the "young" stars, involving dissipation, to the dissipationless "old" stars. The $\beta$ profiles are similar (Fig. 1 of the Letter) and the $\sigma_{\mathrm{p}}$ profiles differ by only $\sim 9 \%$ at $3 R_{\text {eff }}$ (Fig. 2 of the Letter).

A further demonstration is provided in Fig. 5, which compares the results for three merger simulations with the initial gas/baryon fraction ranging from 0 to $70 \%$. In particular, we added to our original sample a case identical to our fiducial Sbc merger ( $52 \%$ gas) but with all the gas particles treated as non-dissipative particles. This "dry" case ends up with a slightly higher $\beta$ (!), which is balanced by a slightly lower $\alpha+\gamma$, resulting in a slightly higher $\sigma_{\mathrm{p}}$.

We conclude that the radial orbits are generic to mergers independent of whether they are "wet" or "dry". The effect of dissipation on $\sigma_{\mathrm{p}}$, via its effect on the density profile, is limited to $\sim 10 \%$, and is therefore of only secondary concern. This indicates that the key results from our 

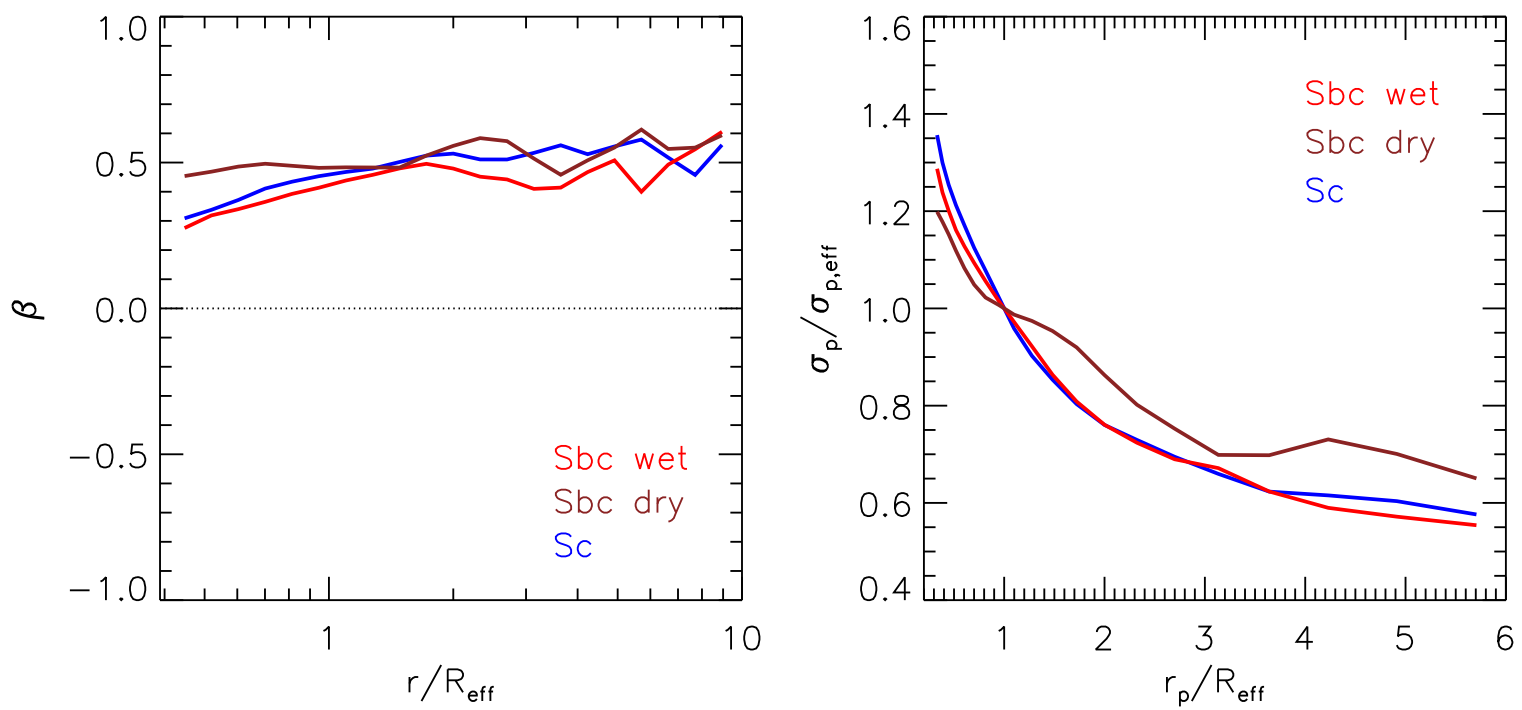

Figure 5. Robustness to dissipation. Anisotropy and velocity dispersion of the "old" stars for mergers with different gas fractions. Three projections at two time steps are stacked at $R_{\text {eff }}$. The red and brown correspond to the same Sbc merger with initially $52 \%$ and zero gas/baryons respectively. The blue is an Sc merger with $70 \%$ initial gas. The effect of dissipation is small, at the level of $\sim 10 \%$ in $\sigma_{\mathrm{p}}$.

merger sample are representative of a variety of mergers, "wet" and "dry", expected in a LCDM universe.

One could argue in passing that the higher $\alpha+\gamma$ resulting from the "wet" mergers, being closer to the observed $\alpha+\gamma$ in the 4 ellipticals discussed (based on Fig. 2 of the Letter), indicates that some degree of dissipation is actually required in order to produce the correct density and velocity profiles of elliptical galaxies. This is, however, only a marginal indication which is worth further study.

The effects of varying the recipes for star formation and feedback on the relevant features addressed in this Letter are yet to be explored.

\section{Robustness to major versus minor mergers}

Our key results are not restricted to 1:1 mergers either. This is demonstrated in Fig. 6, which compares our fiducial 1:1 case with several 1:3 and 1:10 mergers. After a single minor merger, the remnant does not necessarily resemble an elliptical galaxy (e.g. the disk of the big progenitor is still intact), but the halo stars originating in the small progenitor are on very radial orbits, yielding final dispersion profiles which are hardly distinguishable from the 1:1 case. The radial orbits and the low velocity dispersion are thus generic to the stellar haloes of merger remnants, major or minor. This confirms what we heard privately from J. Navarro regarding their preliminary findings based on a cosmological sequence of mergers. It indicates that the key results from our merger sample are representative of a variety of mergers, major and minor, expected in a LCDM universe.

Our methodology for checking the effects of minor mergers is limited by the fact that we have 

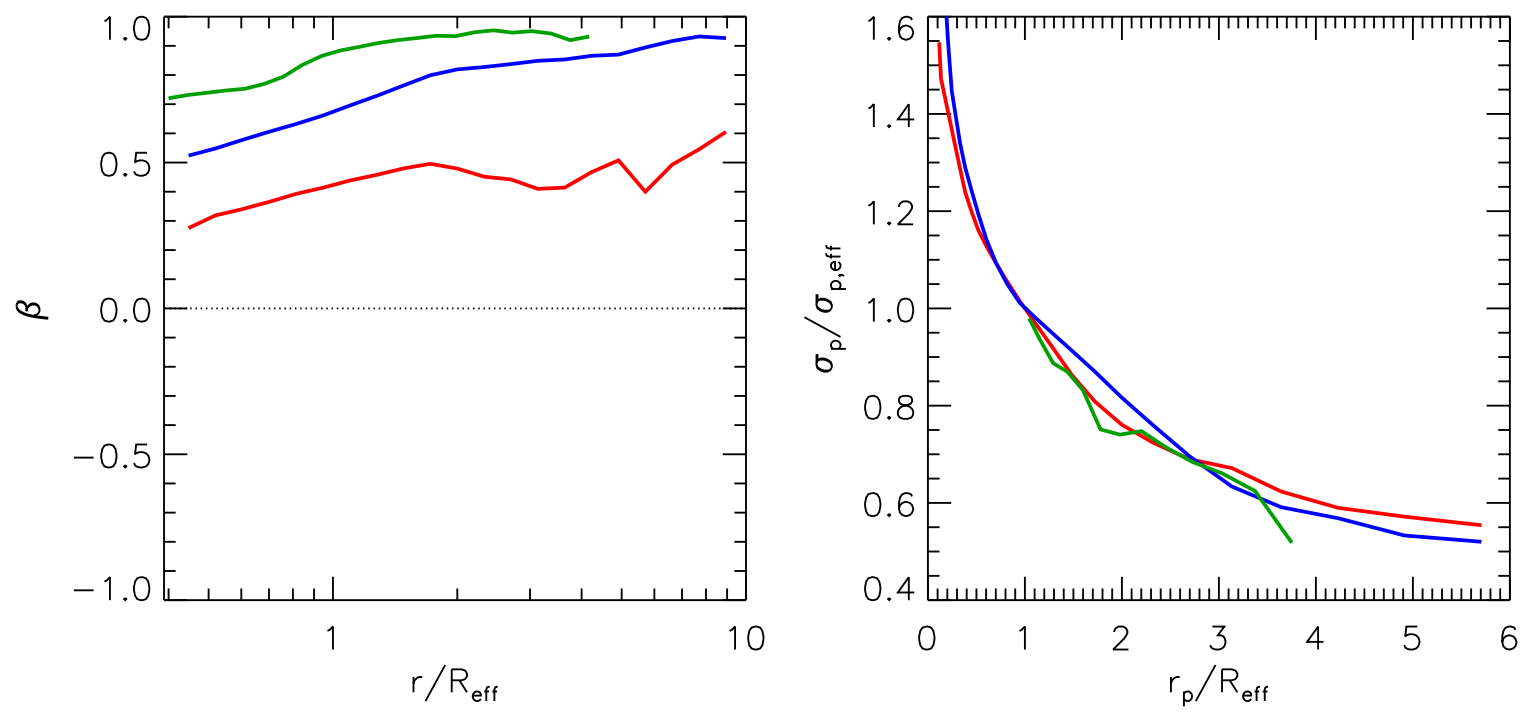

Figure 6. Robustness to major versus minor mergers. Anisotropy and velocity dispersion for major and minor mergers with different mass ratios. For each merger, three projections at two time steps are stacked at $R_{\text {eff }}$. Red: our fiducial 1:1 Sbc merger. Blue: four 1:3 G mergers stacked together. Green: four 1:10 minor mergers stacked together. In the unequal mass mergers, only the "old" stars of the smaller progenitors are used, to better mimic the halo population after a sequence of such mergers and avoid the surviving disk component of the bigger progenitor. The orbits tend to be more radial for the unequal mass mergers, while the final dispersion profiles are hardly distinguishable from the 1:1 case.

performed so far single mergers only. Our logic is that after multiple minor mergers, the final profile is dominated by the many small progenitors which are all contributing to increased radial anisotropy. The detailed study of minor mergers is beyond the scope of this Letter.

\section{Robustness to the presence of a bulge}

The effect of a relatively small bulge is seen in Fig. 7. It reduces $\beta$, but increases the central stellar concentration, together leaving the velocity dispersion hardly changed.

We cannot tell from our current simulations what happens when two ellipticals merge. We may speculate that if $\beta$ turns out to decrease in such mergers (as indicated by the effect of a bulge), while the density profile does not steepen (unlike the case of a bulge), the result may be a slight increase in $\sigma_{\mathrm{p}}$. Given that galaxies in groups are more likely to be ellipticals than in the field, ${ }^{36}$ the dominant group elliptical is more likely to be formed by the mergers of elliptical galaxies (or bulge-rich spirals). If $\beta$ is indeed lower in the corresponding remannts, and if the density profiles are the same as in spiral mergers, one would predict that dominant group members, which are also the most massive ellipticals, should have higher dispersions, as is indirectly observed. ${ }^{16}$ This will be interesting to address in future work, but it is not required for the main point of our current Letter concerning the puzzling low-dispersions in intermediate $\left(L_{*}\right)$ ellipticals. 

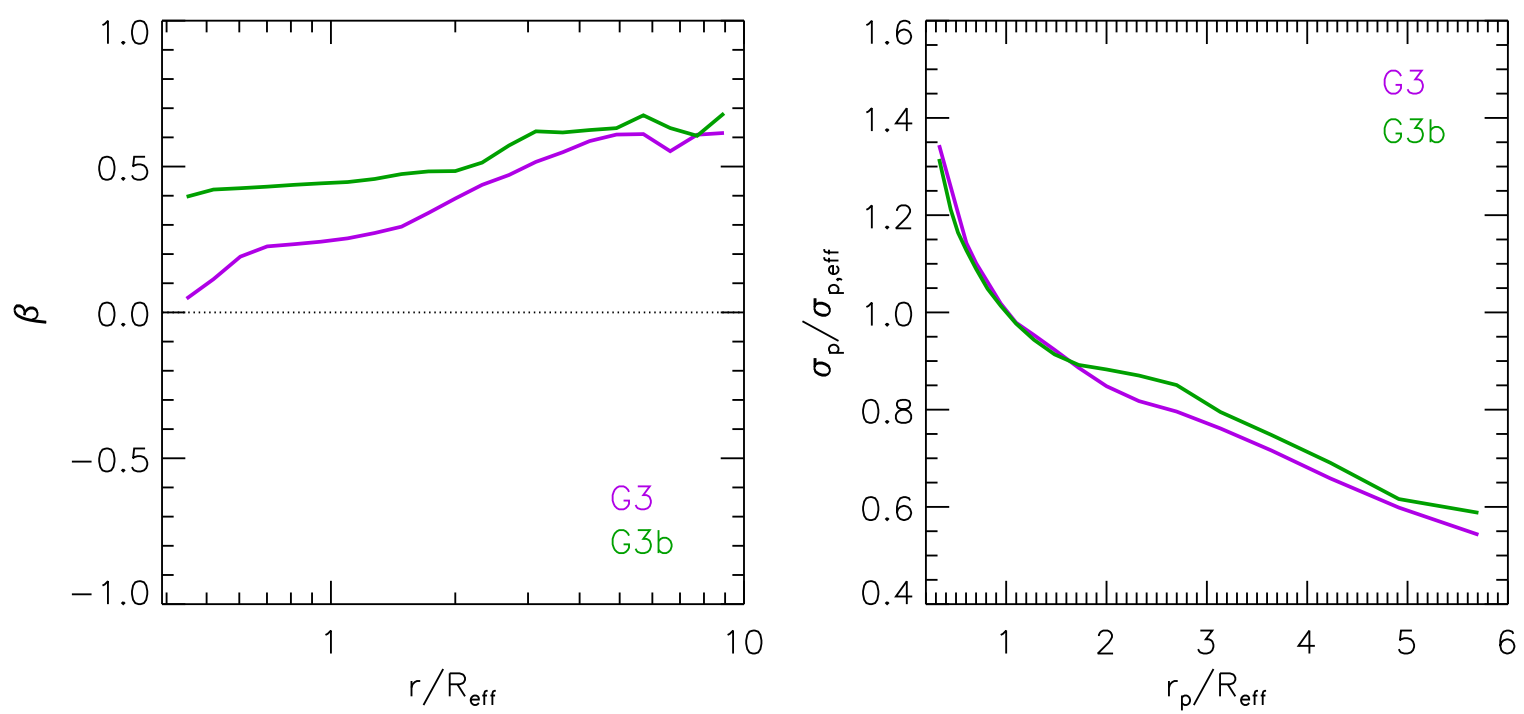

Figure 7. Robustness to the presence of a bulge. Anisotropy and velocity dispersion of the "old" stars for mergers with and without a $22 \%$ bulge component. Three projections at two time steps are stacked at $R_{\text {eff }}$. The purple is a $\mathrm{G}$ merger with $20 \%$ initial gas to stars+gas and $22 \%$ bulge to stellar disk ratio. The green is the same but excluding the bulge.

\section{Long-term stability}

We have continued one of the $\mathrm{G}$ mergers for a longer time, for a total of $6 \mathrm{Gyr}$, where the merger occurred at $\sim 2.5$ Gyr. Shown in Fig. 8 is the time evolution of the profiles of $\beta$ and $\sigma_{\mathrm{p}}$. During the first 0.75 Gyr after the merger (3 dark curves), the system is not yet fully relaxed, though the high $\beta$ and low $\sigma_{\mathrm{p}}$ are already established. During the following 3 Gyr (blue and light-blue curves) the profiles are very stable, at least until 3.5 Gyr after the merger. The slight evolution is limited to central regions where remnant gas is slowly forming more stars.

One can evaluate to what extent the simulated merger remnants obey the Jeans equation, given their deviations from spherical symmetry, variations in time, and possible other deviations from full equilibrium. Given in each remnant our measured values for $\alpha(r), \beta(r), \gamma(r)$ and $\sigma_{r}(r)$, we have used the spherical Jeans equation to estimate the total mass $M_{\text {Jeans }}(r)$ within radius $r$, in comparison with the true $M(r)$. We find near $3 R_{\text {eff }}$ for the 10 remnants at about 1 Gyr after the merger $M_{\text {Jeans }}(r) / M(r) \simeq 0.9 \pm 0.15$. This is one possible measure of the degree of validity of the spherical Jeans equation in these systems.

\section{Parametrizing the velocity-dispersion profiles}

One way to quantify the declining velocity dispersion profile is by fitting a power-law with slope $\gamma$ to $\sigma_{\mathrm{p}}^{2}\left(r_{\mathrm{p}}\right)$ in a given radius range. The slope has been determined for each of the 60 individual profiles by linear regression on equally-log-spaced interpolation points in the range $0.5-6 R_{\text {eff }}$. The mean and standard deviation are $\gamma=0.53 \pm 0.16$ for "all" the stars, and $\gamma=0.61 \pm 0.22$ for 

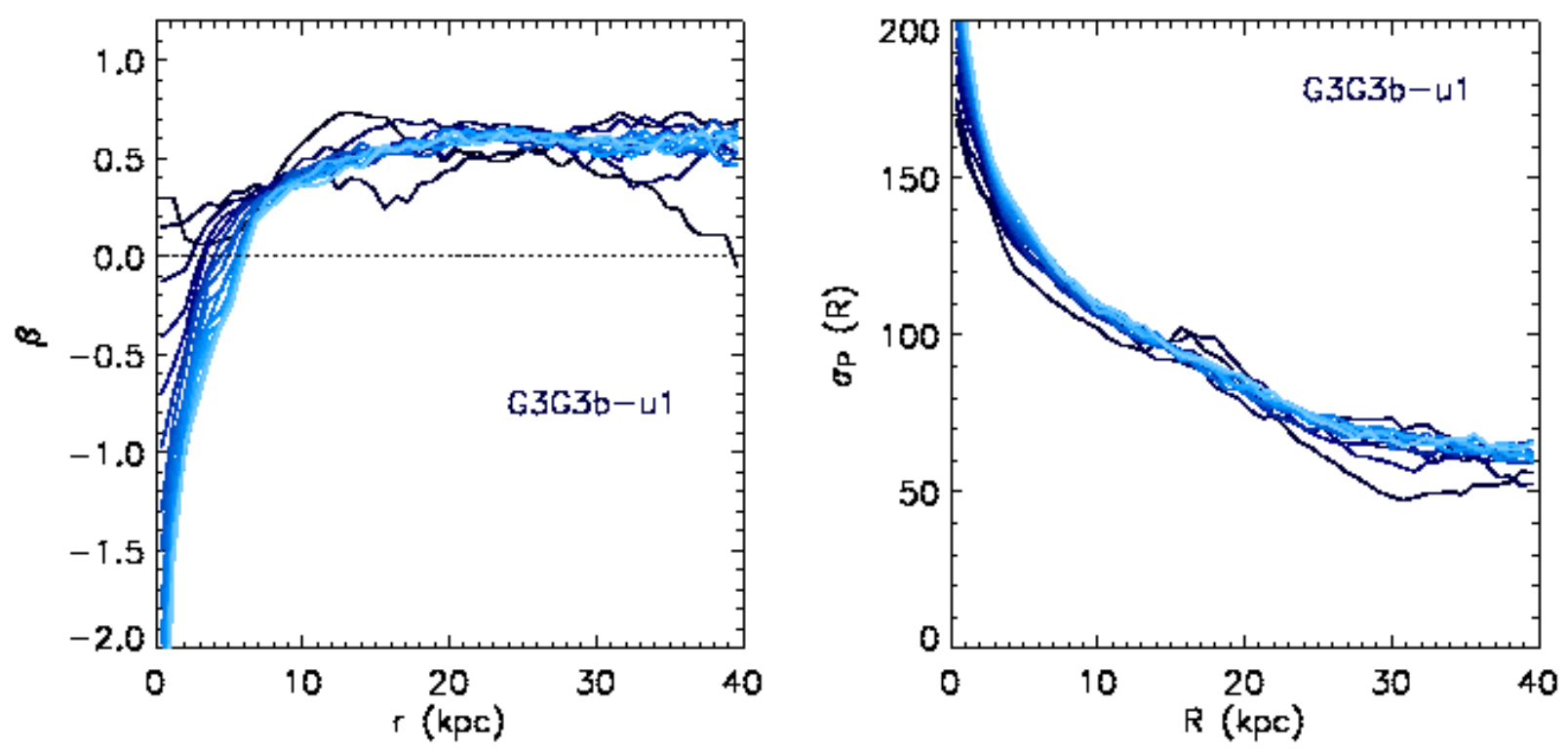

Figure 8. Long-term stability. The evolution of the $\beta$ and $\sigma_{\mathrm{p}}$ profiles in time after the merger $(t=2.5$ Gyr) till $t=6$ Gyr. The curves are plotted at intervals of 0.25 Gyr; they start black (dark blue) and become light blue progressively in time. The profiles are stable at least until 3.5 Gyr after the merger.

the "young" stars. The PN data for the 4 observed galaxies, in the same radius range, give $\gamma_{p}=0.59 \pm 0.08 \pm 0.10$, for the mean, error on the mean (using error propagation), and scatter. There is an agreement between the simulations and the data, both for "all" the stars and for the "young" stars.

The $\sigma_{\mathrm{p}}$ profiles of the four observed galaxies were put together in Fig. 2 of the paper for illustrative purposes. The scaling procedure, explained in Methods, is robust to the exact method of scaling and the range of data used for it. We tried different minimum radii for the stellar data used compared to the fiducial $0.2 R_{\text {eff }}$. The resulting multiplication factors to get $\sigma_{\mathrm{p}}=1$ at $R_{\text {eff }}$ for the 4 galaxies (NGC 4494, 3379, 821, 4697 respectively) are as follows. For $r>0.2 R_{\text {eff }}:(0.00779$, $0.00761,0.00637,0.00714)$, for $r>0.6 R_{\text {eff }}:(0.00758,0.00740,0.00656,0.00708)$, for $r>1.0 R_{\text {eff }}$ : (0.00788, 0.00806, 0.00623, 0.00685). Using the PNs only we obtain: $(0.00804,0.00779,0.00656$, $0.00681)$. The relative changes are of a few percent only.

\section{The full LOSVD and the $h_{4}$ moment}

We find that our sample of merger remnants spans a range of LOSVD's similar to the observed sample, as traced both by stars and by PNs. This is illustrated first in Fig. 9 which shows the LOSVD of three of our merger remnants in comparison with three observed galaxies, including negative and positive kurtosis (or $h_{4}$ ).

We computed the central values of the fourth moment, $h_{4}$, for the stars in the simulated remnants using narrow radial bins, trying to mimic the typical observer's procedure. We made sure that the result within $R_{\text {eff }}$ is insensitive to the actual bin size (differences less than 10\%) once 

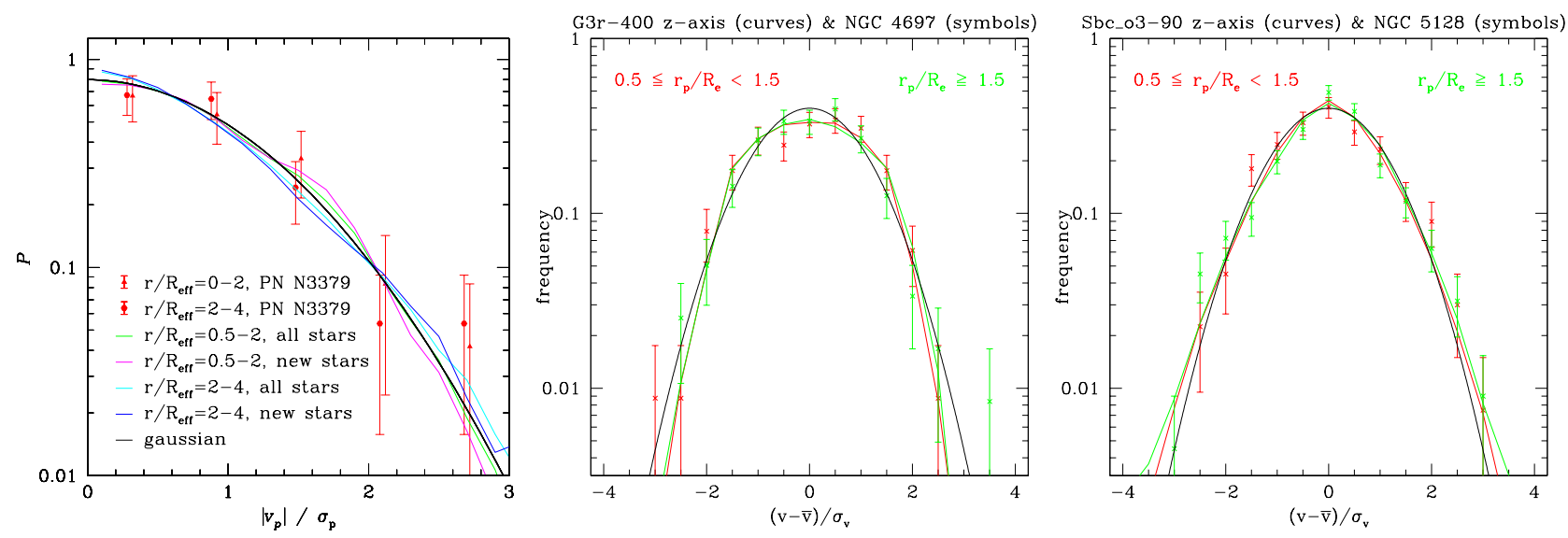

Figure 9. Line-of-sight velocity distribution $P\left(\left|v_{\mathrm{p}}\right| / \sigma_{\mathrm{p}}\right)$ (LOSVD) of simulation versus data. The black curve marks a Gaussian distribution. The LOSVD is computed in two radial bins, as marked, within which the variation of $\sigma_{\mathrm{p}}$ is limited. The data is for N3379, N4697 and N5128 from left to right respectively. The deviations from Gaussian are small, with a tendency for negative and positive kurtosis in N4697 and N5128 respectively. The curves are LOSVD from three simulations. Left: the fiducial Sbc merger, stacked views from three orthogonal directions at the final time, with a near Gaussian LOSVD. Middle: a face-on view of a retrograde merger remnant (G3r) showing a negative kurtosis. Right: a face on view of another Sbc merger remnant showing a positive kurtosis. We learn that a high anisotropy, $\beta \sim 0.5$ beyond $R_{\text {eff }}$, does not necessarily imply strong deviations from a Gaussian distribution.

the bins are significantly narrower than $R_{\text {eff }}$. Fig. 10 shows the profiles of $h_{4}$ for all the simulated remnants stacked together. The most relevant curve refers to the average over many random lines of sights. The central value is $h_{4} \simeq 0.03 \pm 0.05$. The average within $R_{\text {eff }}$ is $h_{4} \simeq 0.02 \pm 0.04$. In the outer range relevant to PNs, $1-4 R_{\text {eff }}$, it is $h_{4} \simeq 0.01 \pm 0.05$. However, more extreme values can be obtained if the line of sight happens to be along one of the principal axes. Along the major axis the central $h_{4}$ could be $\sim 0.1$ or larger, and along the minor axis it could be $\sim-0.7$ at some radii and even more negative.

Comparing the gas-poor $\mathrm{G}$ mergers $(\sim 20 \%$ gas $)$ and the gas-rich Sbc mergers $(\sim 50 \%$ gas $)$ we find similar results for the average $h_{4}$ along random lines of sight, but the G cases tend to show less extreme values when viewed along the major or minor axes, reflecting less important central disk components in these cases, as expected.

These results are consistent with those of other simulations of "dry" mergers, ${ }^{37,38} h_{4}=0.02 \pm$ 0.04 .

The simulated $h_{4}$ is consistent with the observed $h_{4}$ for the integrated stellar light in ellipticals. This includes the typical values ${ }^{39} h_{4} \sim 0.00 \pm 0.04$ and those for the four galaxies of the current Letter. ${ }^{39-41}$

Fig. 10 also shows the $h_{4}$ profile for NGC 3379 as measured by four different observations. Within $0.3 R_{\text {eff }}$ they span the range $h_{4} \simeq 0.0 \pm 0.05$. The SAURON results ${ }^{42}$ are slightly on the high side, ranging from $\sim 0.04$ to $\sim 0.08$. At $r>0.3 R_{\text {eff }}$ the $h_{4}$ measurements are noisier; they 
Major Mergers
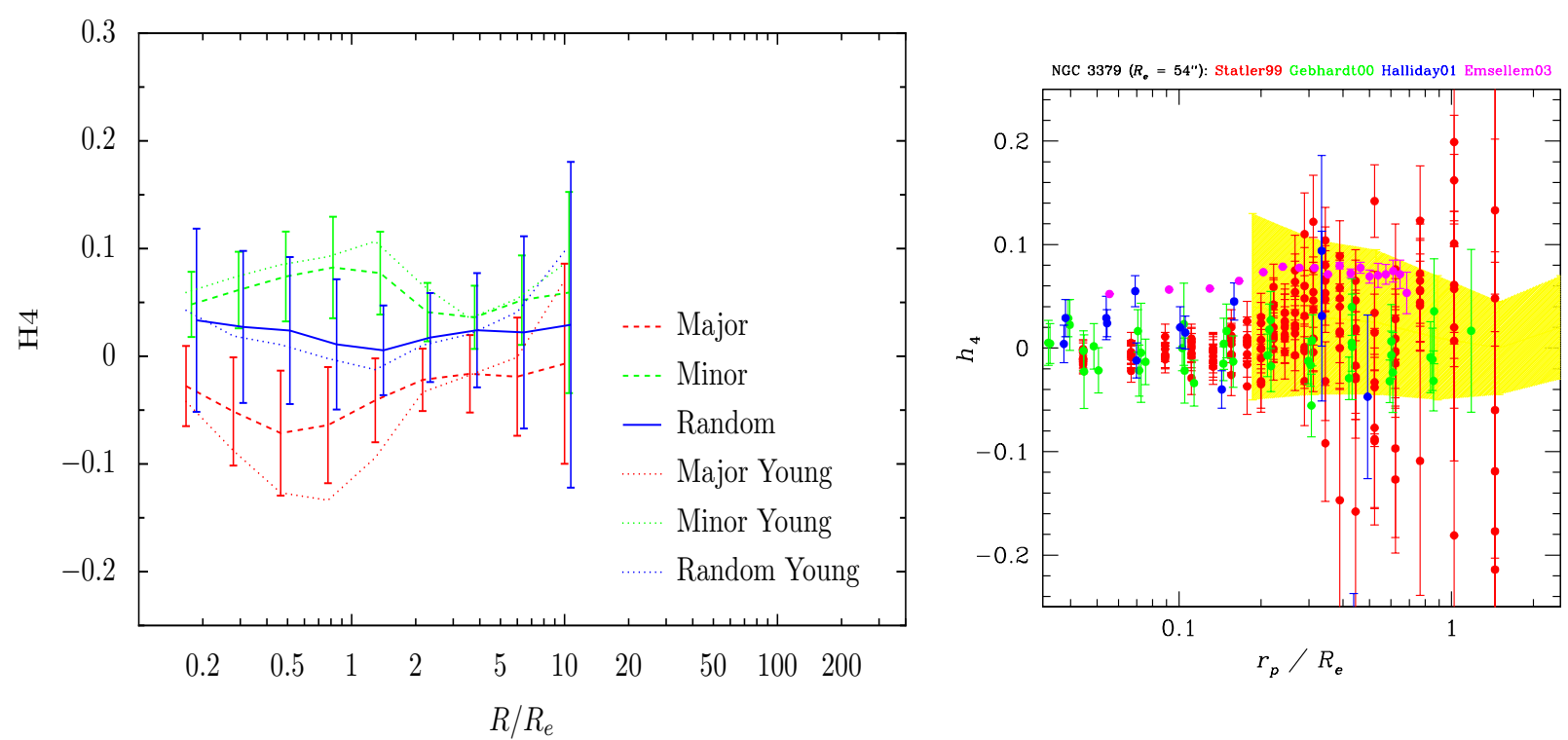

Figure 10. The 4th moment of the LOSVD. Left: Profiles of $h_{4}$ for all simulated remnants stacked together, "all" and "young" stars. The line of sight is either random (blue), or along one of the principal axes of the inertia tensor (as labeled). The error bars mark $1 \sigma$ scatter. Right: Profiles of $h_{4}$ for NGC 3379 from 4 different sources. The $1 \sigma$ range for the "random" view of the simulated remnants is shaded yellow. There is good agreement between the simulations and the observed galaxies.

extend to values above +0.1 and below -0.1 . In SAURON, $h_{4}$ is $0.080-0.085$ when averaged over circular bins, with plenty of azimuthal scatter which may be due poor spectral resolution. The SAURON $h_{4}$ profile of NGC 821 is similar, with $h_{4} \simeq 0.05$ within $R_{\text {eff }}$. The measured $h_{4}$ profiles in these two galaxies are thus consistent with the distribution of $h_{4}$ profiles in the merger remnants.

Despite the general agreement with the observed values of $h_{4}$, we stress that our sample of merger simulations is not supposed to be a fair sample of all the mergers that lead to today's ellipticals. Clearly, not all ellipticals, maybe not even most of them, had recent wet major mergers of disk systems. However, we demonstrated that the key properties relevant to the present Letter, such as the steep density profiles, high $\beta$ and low $\sigma_{\mathrm{p}}$ at large radii, are robust to wet and dry mergers, major and minor. The simulations do not need to be as representative in terms of the inner structure, where the wet mergers tend to lead to a density peak and a small disk, as long as these inner features do not affect the key features at large radii. In turn, we do not use here the simulated properties in the inner regions as a diagnostic for the physics involved in the formation of elliptical galaxies, being dry or wet, etc.

One lesson from our simulations is that a high anisotropy, $\beta \sim 0.5$ beyond $R_{\text {eff }}$, does not necessarily imply strong deviations from a Gaussian distribution. This suggests that the assumed ${ }^{43}$ tight correlation between $h_{4}$ and $\beta$ is model dependent and uncertain in several ways: (a) it is based on the assumptions of spherical symmetry and a specific density profile, (b) it is based on uncertain and limited orbit-library modeling, (c) it assumes that inner stars and outer PNs are 

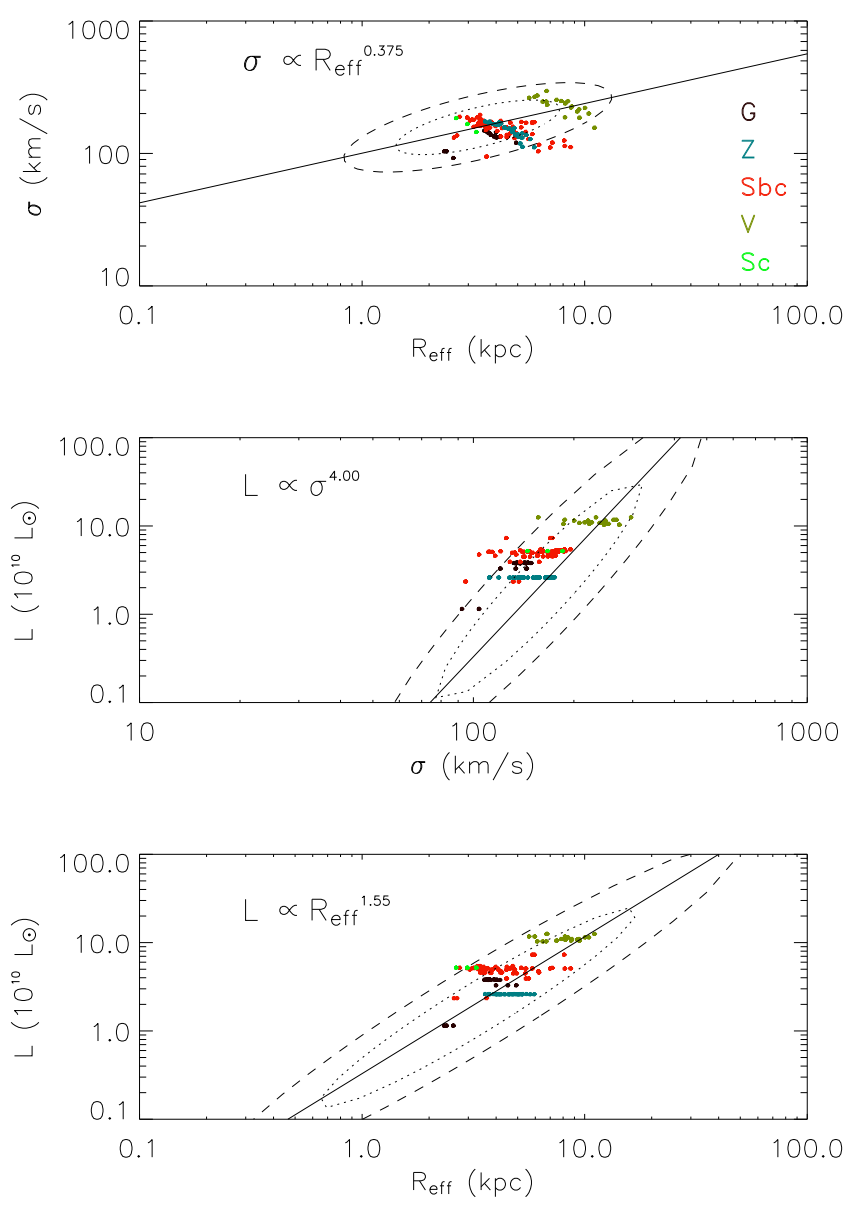

Figure 11. The global structure properties of the simulated merger remnants (symbols, colored by the merger type) in comparison with the Fundamental Plane distribution of elliptical galaxies in SDSS ( $1 \sigma$ and $2 \sigma$ contours). The dispersion velocity $\sigma$ is measured at the central regions. The luminosity is derived from the stellar mass assuming an effective $M / L=3$.

the same population, and (d) $h_{4}$ is also related to rotation. We therefore do not draw conclusions from the predictions of $\beta$ based on $h_{4}$.

\section{Matching other properties of ellipticals}

Fundamental Plane. The merger remnants qualitatively agree with the Fundamental Plane of elliptical galaxies. This is demonstrated in Fig. 11. The deviations are typically at the $1 \sigma$ level. This is work in preparation.

Surface-brightness profile. The simulated stellar surface-brightness profiles resemble the de Vaucouleurs profile remarkably well from below $R_{\text {eff }}$ to several $R_{\text {eff }}$ (Top panel of Fig. 2 of the Letter). Some of our simulations produce a central stellar excess $(<1 \mathrm{kpc})$ due to gas settling in an inner disk at the remnant center (which might be prevented once AGNs are included). This 
explains the negative values of $\beta$ at small radii in many of the simulations. Such an excess is not seen in typical ellipticals, but is seen in observed mergers. ${ }^{44,45}$ This inner excess does not seem to affect the kinematics of the relevant, loosely bound stars beyond $R_{\text {eff }}$. The line-of-sight velocity dispersions are not directly affected by the properties of the material at smaller radii. Indeed, we find no correlation between the inner excess and the anisotropy of the halo orbits. The small excess is more pronounced in the wet Sbc mergers than in the dryer G mergers, and more so for the "young" stars than the "old" ones. The fact that the low $\sigma_{\mathrm{p}}$ and steep $\gamma$ are also seen for "all" the stars and for the $G$ mergers indicates that the central excess has no important effect on our results.

Ellipticity. The remnants of the 1:1 mergers provide projected axial ratios near $R_{\text {eff }}$ that typically range from 1:1 to 1:2, not unlike observed ellipticals. In 3D, the median configuration is 1:0.95:0.5, namely oblate, but there are triaxial and prolate cases as well, in the ballpark of most observational findings. ${ }^{46-49}$ Retrograde mergers are naturally somewhat rounder than the more aligned prograde mergers.

Rotation. The simulated remnants may have a rotating gas-dominated disk at their centers, but they are typically far from rotational support at $R_{\text {eff }}$ and beyond. For example, our fiducial Sbc case has near $R_{\text {eff }}$ an axial ratio $c / a \simeq 0.5$ (or $\epsilon \simeq 0.5$ ). The edge-on view gives a maximum of $V / \sigma_{0} \simeq 0.25$ at $1.5 R_{\text {eff }}$, well within the range of the estimates for the 4 observed ellipticals: $V / \sigma_{0}=0.15-0.29$ (A. Romanowsky, private communication). An isotropic case would have given $V / \sigma_{0}=[\epsilon /(1-\epsilon)]^{1 / 2} \sim 1$. Then $\left(V / \sigma_{0}\right)^{*}=\left(V / \sigma_{0}\right) /\left(V / \sigma_{0}\right.$,isotropic $) \sim 0.25$, namely, the system ellipticity is dominated by the triaxiality of the velocity dispersion tensor, not by rotation (consistent with non-dissipative merger simulations ${ }^{50}$ ).

In another merger simulation ${ }^{51}$ the remnant looks like a spiral almost immediately after the merger. This is perhaps due to their somewhat pathological orbit, where the spins and angular momenta are aligned. It is true that our Sbc merger remnants have certain disk-like qualities because they started out with a high gas fraction and an extended gas disk. However, the $\mathrm{G}$ remnants have less disks in them, implying that the high $\beta$ and low $\sigma_{\mathrm{p}}$ are not a result of the diskiness of the remnant.

Yet another set of merger simulations ${ }^{52}$ indicates a tendency for more tangential orbits at large radii. This is likely to be due to their wide angle and slow orbits, in which the tidal effects at pericenter are weaker than in our more radial and faster collisions.

Dark-matter density profile. The simulated DM profiles in the relevant range between $R_{\text {eff }}$ and a few $R_{\text {eff }}$ are typically of log slope slightly flatter than -2 . This is in the ballpark of what might be expected from CDM haloes below $\sim 0.1 R_{\text {vir }}$ subject to the baryonic dissipative effects, ${ }^{26}$ as mentioned in the text. (This could be compared to the unperturbed NFW profile of haloes in DM-only simulations, which flattens to $\sim-1.5$ near $R_{\text {eff }}$ )

\section{$9 \quad$ Scatter induced by triaxiality}

Fig. 3 illustrates the triaxial shape of our typical merger remnant. Fig. 12 demonstrates the effects of triaxiality on the velocity dispersion profile. 


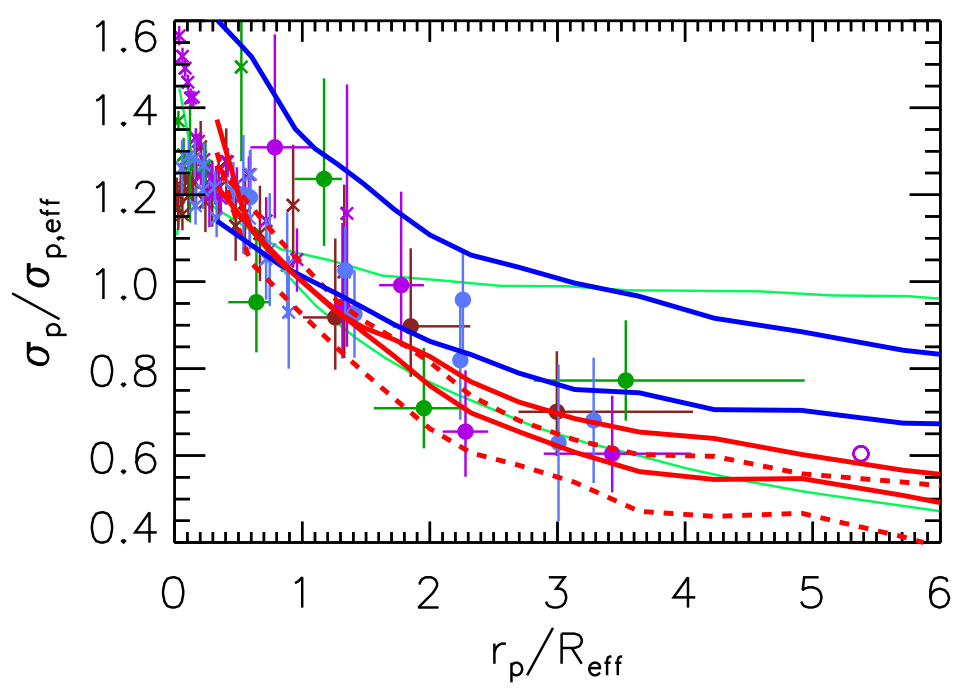

Figure 12. Effect of triaxiality and rotation. Projected velocity dispersion in the Sbc merger remnant of Fig. 3 as viewed "face-on" (lower) and "edge-on" (upper). Notation as in Fig. 2 of the Letter. The triaxiality in the velocity dispersion tensor leads to a $\sim 20 \%$ difference between the two projection directions. The $\sigma_{\mathrm{p}}$ profiles from both views of "all" the stars are consistent with the observed $\sigma_{\mathrm{p}}$. The edge-on view of the "young" stars is also in good agreement with the observed $\sigma_{\mathrm{p}}$, while the face-on $\sigma_{\mathrm{p}}$ generally lies below the data. While rotation is part of the anisotropy of the tangential component, the simulated system is not supported by rotation: its edge-on rotation velocity is only $\simeq 25 \%$ of the circular velocity, with $V / \sigma_{0} \simeq 0.2$ at $\gtrsim R_{\text {eff }}$, and with $V / \sigma \simeq 0.3$ below $R_{\text {eff }}$ rising to $V / \sigma \simeq 0.5$ beyond $2 R_{\text {eff }}$.

While the four ellipticals addressed here have low, declining velocity dispersion profiles, there are other ellipticals which show higher or flatter dispersion profiles. As mention in the text, such profiles may be the result of: (a) a specific projection angle in a triaxial or rotating system, (b) a high angular-momentum merger, (c) a retrograde merger, (d) a merger with less dissipation, or (e) probing with tracers different from PNs, such as globular clusters (of a flatter density profile and lower anisotropy). The high $\sigma_{\mathrm{p}}$ cases may be associated with more massive galaxies and with centers of groups - a subject of future work.

\section{On the PN age}

One may be concerned about the validity of the assertion that the bright PNs are likely to be younger than 3 Gyr based on the theoretical work of Marigo et al.. ${ }^{30}$

We should stress first that the issue of whether the PNs are young or not is secondary to our results, corresponding to only a small additional reduction of less than $10 \%$ in $\sigma_{\mathrm{p}}$. This is smaller than the uncertainties and variation among the galaxies, and smaller than the variations due to the merger-orbit parameters or projection direction. Our key result, of radial anisotropy, is even more robust, as seen in Fig. 1 of the Letter and as demonstrated above. 
Still, the theoretical analysis seems straightforward and we are not aware of convincing theoretical arguments against its validity. The similar luminosity function of PNs in different ellipticals is an empirical result which should rather be explained (e.g. by a similar merger history for these galaxies, including many minor mergers), but it does not seem to justify ignoring the theoretical predictions based on stellar theory. It is therefore fair to consider the PN young age as a possibility.

We may comment in passing that the hypothesis that the PNs are blue stragglers produced by stellar collisions, may perhaps work for globular clusters, but it is hard to see how the stellar collision rate could be high enough at the outskirts of elliptical galaxies. Perhaps a recent merger would help... The idea of blue stragglers may work if they are produced by mass transfer in common binary systems. ${ }^{53}$

\section{Rising versus constant $\beta$}

Is the gradual rise in $\beta(r)$ responsible for a steeper decline in $\sigma_{\mathrm{p}}$ ? This seems consistent with the trend seen in the comparison of "dry" and "wet" simulations in Fig. 5, for which $\beta$ is a constant and rising respectively.

However, a test using the spherical Jeans equation indicates that low $\beta$ values below $R_{\text {eff }}$ have negligible effects on $\sigma_{\mathrm{p}}$ beyond $R_{\text {eff }}$. Fig. 13 compares two solutions of the Jeans equation, one with $\beta=0.5$ at all radii, and the other with $\beta=0$ at small radii, smoothly rising near $R_{\text {eff }}$ to $\beta=0.5$ at large radii following an assumed functional form. ${ }^{21}$ For example, $\beta=0.25$ at $1.4 R_{\text {eff }}$. The shape of the $\beta$ profile is similar to the average shape in our simulated remnants. After matching the solutions at $R_{\text {eff }}$, the $\sigma_{\mathrm{p}}$ profiles are almost identical at all larger radii. Based on this example, we suspect that the rising nature of the profile may not to be the key factor driving the decline in $\sigma_{\mathrm{p}}$.

We recently learned from A. Romanowsky (private comm.) about the $\beta$ profile in their preferred orbit-library solution to N3379: it has a negative value of $\beta=-0.3$ at $R_{\text {eff }}$, and it rises toward $\sim+0.5$ but only beyond $5 R_{\text {eff }}$. While being rising in part of the relevant range, it does not seem to reach high enough values there, and therefore does not support a massive DM halo. In comparison, in many of our simulations, $\beta$ is positive at $R_{\text {eff }}$, it is close to 0.5 already at $2 R_{\text {eff }}$, and it keeps rising toward larger radii.

\section{Why was the DM solution missed before?}

Using merger simulations, we provide "a" fully specified solution, consistent with what we know about DM haloes in other galaxies and in the $\Lambda$ CDM scenario, which seems not to violate all the relevant observed aspects within the given uncertainties. Independent of whether this solution is indeed "the" solution for elliptical galaxies, it is claimed ${ }^{4}$ (R03) to have been missed in the original application of the orbit-library maximum-likelihood algorithm.

We cannot provide a definite answer to why this solution was missed; the published material (as well as private communication with the authors) provided only partial information for evaluating the method or for fully characterizing their preferred solutions. A sample output from our simulations can provide a useful testbed for their method. 


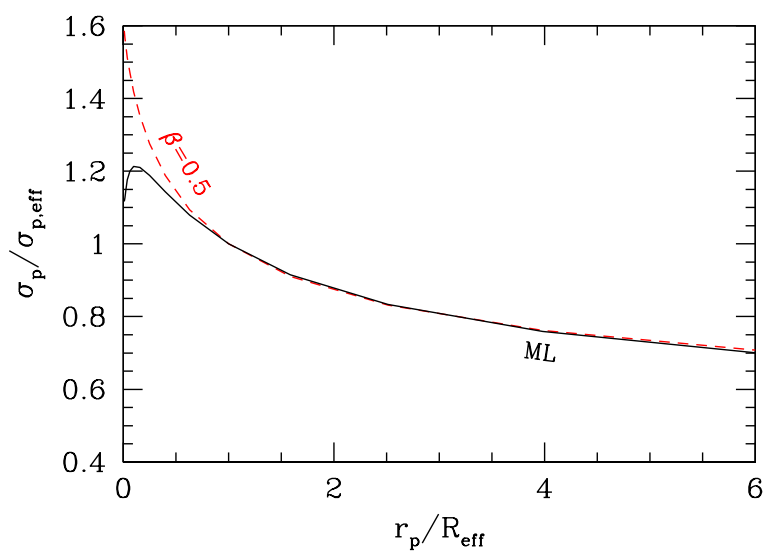

Figure 13. Rising versus constant $\beta$. A comparison of solutions to the spherical Jeans equation; one with $\beta=0.5$ at all radii and the other with a rising $\beta(r)$ from zero at small radii to 0.5 at large radii, with the transition near $R_{\text {eff }}$.

However, it is not at all surprising that a complex maximum-likelihood method based on an orbit library may miss some of the solutions. We can only raise several ideas for where such method could go wrong, as follows:

1. Assuming a stationary potential. The outer system may still be evolving (see Fig. 4). We find in our Sbc simulations that the Jeans equation is typically valid to $\sim 10 \%$ accuracy, in the sense that the total mass within $1-3 R_{\text {eff }}$ as derived by the Jeans equation is $\sim 90 \%$ of the true value. In a few cases the deviation can be larger.

2. Assuming spherical symmetry. Ellipticals can appear circular in one projection while actually having an axial ratio of 1:2 (see Fig. 3 ).

3. Assuming density profiles with a specific, limited shape, such as NFW for the DM. There is no reason to believe that the DM haloes in ellipticals, under the influence of dissipation within them, still obey their dissipationless NFW shape near $R_{\text {eff }}{ }^{26}$ In fact, our simulations demonstrate that a major merger of NFW haloes including disks may lead to slight deviations from NFW in the relevant range (see Fig. 1 of the Letter, showing a power-law of slope $\sim-2$ in the relevant range).

4. Their orbit-library method heavily depends on constraints from the values of higher velocity moments, such as $h_{4}$, as determined from the central stellar diffuse light. These constraints may be misleading if the PNs detected at large radii actually represent a population that is kinematically different from the central stars.

5. The problem may lie in some incompleteness of the orbit library used, or in the way by which it is composed for best fit. There were claims in the literature about failure to converge when the number of orbits is increased. ${ }^{18,54,55}$ One may suspect that the R03 method is subject to such problems.

6. A multi-parameter (or "non-parametric") maximum-likelihood method may easily miss some of the solutions. 


\section{Robustness and uniqueness of the results}

Unfortunately, full exact predictions of the $\Lambda$ CDM scenario are beyond what can be achieved by current methods. We therefore use state-of-the-art simulations to study in detail a sample of merger cases. This sample, based on plausible assumptions, provides DM-dominated systems which are consistent with the observed ellipticals in all relevant testable respects, and it reproduces the puzzling low velocity dispersions and their variations among different galaxies. This by itself is an important step forward, given that previous work failed to find such a solution and thus challenged the whole cosmological scenario. Demonstrating that the low velocities are not necessarily fatal for $\Lambda \mathrm{CDM}$ is a step forward by itself.

However, the variety of cases simulated provide reasonably compelling evidence for the robustness of the key phenomenon - radial orbits - indicating that this is indeed a generic outcome of a large class of mergers in CDM-like scenarios. In particular, we have demonstrated robustness to dissipation, mass ratio, the presence of a bulge and several other merger characteristics, and have gained insight into the generic origin of the effect via tidal processes.

An alternative solution has been proposed, ${ }^{16}$ appealing to a low halo concentration parameter $C$. Using our Jeans-equation algorithm, we find that even a $C$ as small as $30 \%$ of the mean $\Lambda \mathrm{CDM}$ value fails to fit the $\sigma_{\mathrm{p}}$ profile of NGC 3379 unless $\beta \sim 0.5$ near $R_{\text {eff }}$ and beyond. The fit proposed ${ }^{16}$ requires an unrealistic $C$, five times smaller than the $\Lambda$ CDM value. This indicates that our solution, based on radial stellar orbits, is the only viable solution proposed so far in the literature within the $\Lambda \mathrm{CDM}$ cosmology.

We have demonstrated that the solutions emerging from our sample merger simulations are representative of a large class of mergers likely to occur in a $\Lambda$ CDM cosmology. We conclude that the radial anisotropies necessary for compatibility with the low $\sigma_{\mathrm{p}}$ observed are not really "pathological" but generally expected. Then, triaxiality of the galaxies could play a major role in observing low dispersions; recent star formation could in rare cases also contribute." 


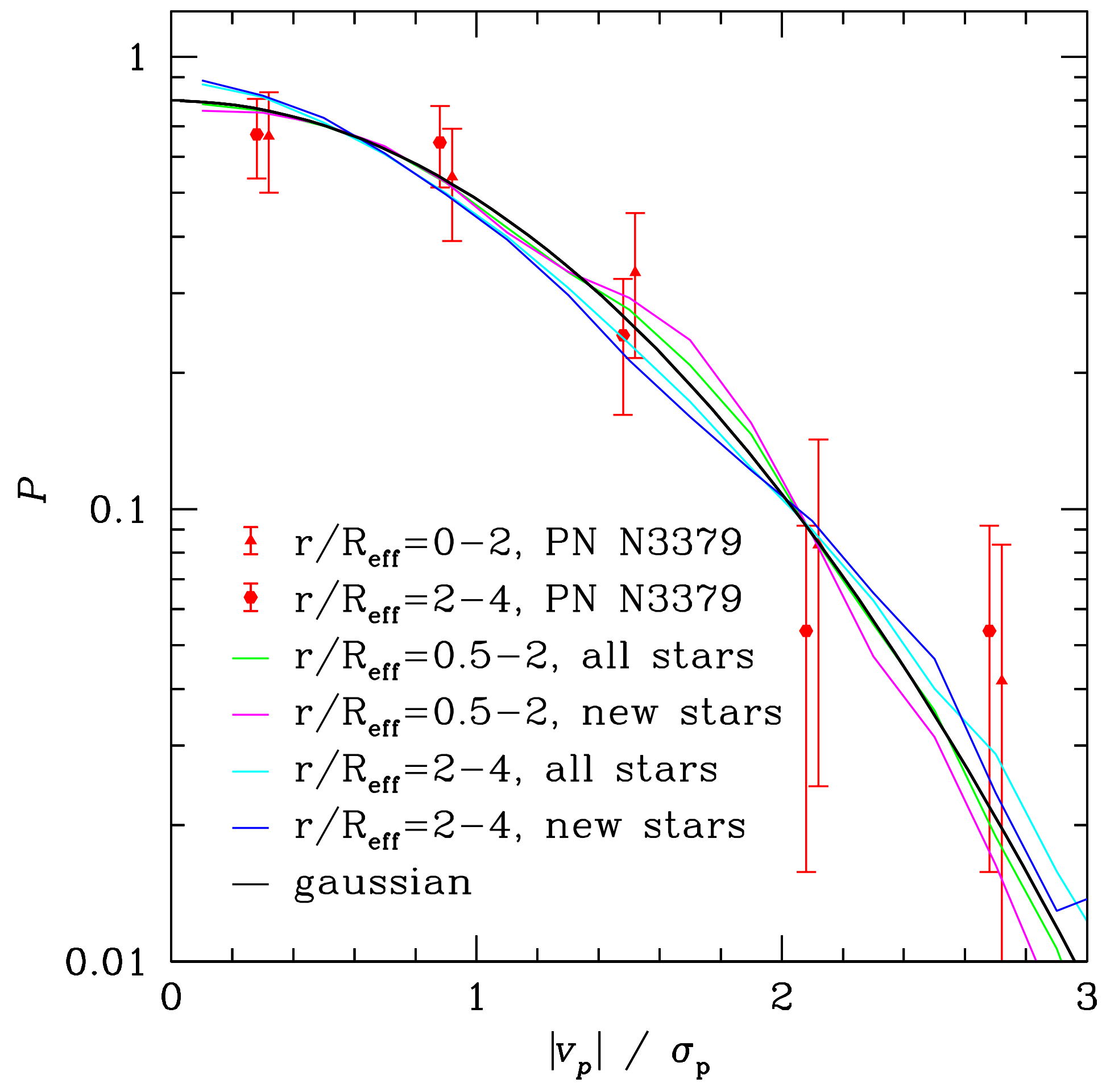

